\title{
Throughput-Optimal Opportunistic Scheduling in the Presence of Flow-Level Dynamics
}

\author{
Shihuan Liu \\ ECE \\ Iowa State University \\ liush08@iastate.edu;
}

\author{
Lei Ying \\ ECE \\ Iowa State University \\ leiying@iastate.edu
}

\author{
R. Srikant \\ ECE and CSL \\ University of Illinois \\ rsrikant@illinois.edu
}

\begin{abstract}
We consider multiuser scheduling in wireless networks with channel variations and flow-level dynamics. Recently, it has been shown that the MaxWeight algorithm, which is throughput-optimal in networks with a fixed number users, fails to achieve the maximum throughput in the presence of flowlevel dynamics. In this paper, we propose a new algorithm, called workload-based scheduling with learning, which is provably throughput-optimal, requires no prior knowledge of channels and user demands, and performs significantly better than previously suggested algorithms.
\end{abstract}

\section{INTRODUCTION}

Multiuser scheduling is one of the core challenges in wireless communications. Due to channel fading and wireless interference, scheduling algorithms need to dynamically allocate resources based on both the demands of the users and the channel states to maximize network throughput. The celebrated MaxWeight algorithm developed in [2] for exploiting channel variations works as follows. Consider a network with a single base station and $n$ users, and further assume that the base station can transmit to only one user in each time slot. The MaxWeight algorithm computes the product of the queue length and current channel rate for each user, and chooses to transmit to that user which has the largest product; ties can be broken arbitrarily. The throughput-optimality property of the MaxWeight algorithm was first established in [2], and the results were later extended to more general channel and arrival models in [3]-[5]. The MaxWeight algorithm should be contrasted with other opportunistic scheduling such as [6], [7] which exploit channel variations to allocate resources fairly assuming continuously backlogged users, but which are not throughput-optimal when the users are not continuously backlogged.

While the results in [2]-[4] demonstrate the power of MaxWeight-based algorithms, they were obtained under the assumptions that the number of users in the network is fixed and the traffic flow generated by each user is long-lived, i.e., each user continually injects new bits into the network. However, practical networks have flow-level dynamics: users arrive to transmit data and leave the network after the data are fully transmitted. In a recent paper [1], the authors show that the MaxWeight algorithm is in fact not throughput optimal in networks with flow-level dynamics by providing a clever example showing the instability of the MaxWeight scheduling. The intuition is as follows: if a long-lived flow does not receive enough service, its backlog builds up, which forces the MaxWeight scheduler to allocate more service to the flow. This interaction between user backlogs and scheduling guarantees the correctness of the resource allocation. However, if a flow has only a finite number of bits, its backlog does not build up over time and it is possible for the MaxWeight to stop serving such a flow and thus, the flow may stay in the network forever. Thus, in a network where finite-size flows continue to arrive, the number of flows in the network could increase to infinity. One may wonder why flow-level instability is important since, in real networks, base stations limit the number of simultaneously active flows in the network by rejecting new flows when the number of existing flows reaches a threshold. The reason is that, if a network model without such upper limits is unstable in the sense that the number of flows grows unbounded, then the corresponding real network with an upper limit on the number of flows will experience high flow blocking rates. This fact is demonstrated in our simulations later.

In [1], the authors address this instability issue of MaxWeight-based algorithms, and establish necessary and sufficient conditions for the stability of networks with flowlevel dynamics. The authors also propose throughput-optimal scheduling algorithms. However, as the authors mention in [1], the proposed algorithms require prior knowledge of channel distribution and traffic distribution, which is difficult and sometimes impossible to obtain in practical systems, and further, the performance of the proposed algorithms is also not ideal.

Since flow arrivals and departures are common in reality, we are interested in developing practical scheduling algorithms that are throughput-optimal under flow-level dynamics. We consider a wireless system with a single base station and multiple users (flows). The network contains both long-lived flows, which keep injecting bits into the network, and shortlived flows, which have a finite number of bits to transmit. The main contributions of this paper include the following:

- We obtain the necessary conditions for flow-level stability of networks with both long-lived flows and short-lived flows. This generalizes the result in [1], where only shortlived flows are considered.

- We propose a simple algorithm for networks with shortlived flows only. Under this algorithm, each flow keeps track of the best channel condition that it has seen so far. 
Each flow whose current channel condition is equal to the best channel condition that it has seen during its lifetime is eligible for transmission. It is shown that an algorithm which uniformly and randomly chooses a flow from this set of eligible flows for transmission is throughputoptimal. Note that the algorithm is a purely opportunistic algorithm in that it selects users for transmission when they are in the best channel state that they have seen so far, without considering their backlogs.

- Based on an optimization framework, we propose to use the estimated workload, the number of time slots required to transmit the remainder of a flow based on the best channel condition seen by the flow so far, to measure the backlog of short-lived flows. By comparing this short-lived flow backlog to the queue lengths and channel conditions of the long-lived flows, we develop a new algorithm, named workload-based scheduling with learning, which is throughput-optimal under flow-level dynamics. The term "learning" refers to the fact that the algorithm learns the best channel condition for each shortloved flow and attempts to transmit when the channel condition is the best.

- We use simulations to evaluate the performance of the proposed scheduling algorithm, and observe that the workload-based scheduling with learning performs significantly better than the MaxWeight scheduling in various settings.

The terminology of long-lived and short-lived flows above has to be interpreted carefully in practical situations. In practice, each flow has a finite size and thus, all flows eventually will leave the system if they receive sufficient service. Thus, all flows are short-lived flows in reality. Our results suggest that transmitting to users who are individually in their best estimated channel state so far is thus, throughput optimal. On the other hand, it is also well known that real network traffic consists of many flows with only a few packets and a few flows with a huge number of packets. If one considers the time scales required to serve the small-sized flows, the large-sized flows will appear to be long-lived (i.e., persistent forever) in the terminology above. Thus, if one is interested in performance over short time-scales, an algorithm which considers flows with a very large number of packets as being long-lived may lead to better performance and hence, we consider the more general model which consists of both short-lived flows longlived flows. Our simulations later confirm the fact that the algorithm which treats some flows are being long-lived leads to better performance although through-optimality does not require such a model. In addition, long-lived flows partially capture the scenario where all bits from a flow do not arrive at the base station all at once. This fact is also exploited in our simulation experiments.

\section{BASIC MODEL}

Network Model: We consider a discrete-time wireless downlink network with a single base station and many flows (users). The base station can serve only one flow at a time.
Traffic Model: The network consists of the following two types of flows:

- Long-lived flows: Long-lived flows are traffic streams that are always in the network and continually generate bits to be transmitted.

- Short-lived flows: Short-lived flows are flows that have a finite number of bits to transmit. A short-lived flow enters the network at a certain time, and leaves the system after all bits are transmitted.

We assume that the set of long-lived flows is fixed, and shortlived flows arrive and depart. We let $l$ be the index for longlived flows, $\mathcal{L}$ be the set of long-lived flows, and $L$ be the number of long-lived flows, i.e., $L=|\mathcal{L}|$. Furthermore, we let $X_{l}(t)$ be the number of new bits injected by long-lived flow $l$ in time slot $t$, where $X_{l}(t)$ is a discrete random variable with finite support, and independently and identically distributed (i.i.d.) across time slots. We also assume $\mathbf{E}\left[X_{l}(t)\right]=x_{l}$ and $X_{l}(t) \leq X^{\max }$ for all $l$ and $t$.

Similarly, we let $i$ be the index for short-lived flows, $\mathcal{I}(t)$ be the set of short-lived flows in the network at time $t$, and $I(t)$ be the number of short-lived flows at time $t$, i.e., $I(t)=|\mathcal{I}(t)|$. We denote by $f_{i}$ the size (total number of bits) of short-lived flow $i$, and assume $f_{i} \leq F^{\text {max }}$ for all $i$.

It is important to note that we allow different short-lived flows to have different maximum link rates. A careful consideration of our proofs will show the reader that the learning algorithm is not necessary if all users have the same maximum rate and that one can simply transmit to the user with the best channel state if it is assumed that all users have the same maximum rate. However, we do not believe that this is a very realistic scenario since SNR variations will dictate different maximum rates for different users.

Residual Size and Queue Length: For a short-lived flow $i$, let $Q_{i}(t)$ which we call the residual size, denote the number of bits still remaining in the system at time $t$. For a long-lived flow $l$, let $Q_{l}(t)$ denote the number of bits stored at the queue at the base station.

Channel Model: There is a wireless link between each flow and the base station. Denote by $R_{i}(t)$ the state of the link between short-lived flow $i$ and the base station at time $t$ (i.e., the maximum rate at which the base station can transmit to short-lived flow $i$ at time $t$ ), and $R_{l}(t)$ the state of the link between long-lived flow $l$ and the base station at time $t$. We assume that $R_{i}(t)$ and $R_{l}(t)$ are discrete random variables with finite support. Define $R_{i}^{\max }$ and $R_{l}^{\max }$ to be the largest values that these random variables can take, i.e., $P\left(R_{j}(t)>\right.$ $\left.R_{j}^{\max }\right)=0$ for each $j \in \mathcal{L} \bigcup\left(\bigcup_{t} \mathcal{I}(t)\right)$. Further, assume that there exist $p_{s}^{\max }$ and $R^{\max }$ such that

$$
\begin{aligned}
& \operatorname{Pr}\left(R_{i}(t)=R_{i}^{\max }\right) \geq p_{s}^{\max } \quad \forall i, t \\
& \max \left\{\max _{i} R_{i}^{\max }, \max _{l} R_{l}^{\max }\right\} \leq R^{\max } .
\end{aligned}
$$

The states of wireless links are assumed to be independent across flows and time slots (but not necessarily identically distributed across flows). The independence assumption across time slots can be relaxed easily but at the cost of more complicated proofs. 


\section{WORKLOAD-BASED SCHEDULING WITH LEARNING}

In this section, we introduce a new scheduling algorithm called Workload-based Scheduling with Learning (WSL).

Workload-based Scheduling with Learning: For a shortlived flow $i$, we define

$$
\tilde{R}_{i}^{\max }(t)=\max _{\max \left\{t-D, b_{i}\right\} \leq s \leq t} R_{i}(s),
$$

where $b_{i}$ is the time short-lived flow $i$ joins the network and $D>0$ is called the learning period. A key component of this algorithm is to use $R_{i}^{\max }$ to evaluate the workload of shortlived flows (the reason will be explained in a detail in Section V]. However, $R_{i}^{\max }$ is in general unknown, so the scheduling algorithm uses $\tilde{R}_{i}^{\max }(t)$ as an estimate of $R_{i}^{\max }$.

During each time slot, the base station first checks the following inequality:

$$
\alpha \sum_{i \in \mathcal{I}(t)}\left\lceil\frac{Q_{i}(t)}{\tilde{R}_{i}^{\max }(t)}\right\rceil>\max _{l \in \mathcal{L}} Q_{l}(t) R_{l}(t),
$$

where $\alpha>0$.

- If inequality (1) holds, then the base station serves a shortlived flow as follows: if at least one short-lived flow (say flow $i$ ) satisfies $R_{i}(t) \geq Q_{i}(t)$ or $R_{i}(t)=\tilde{R}_{i}^{\max }(t)$, then the base station selects such a flow for transmission (ties are broken according to a good tie-breaking rule, which is defined at the end of this algorithm); otherwise, the base station picks an arbitrary short-lived flow to serve.

- If inequality (1) does not hold, then the base station serves a long-lived flow $l^{*}$ such that

$$
l^{*} \in \arg \max _{l \in \mathcal{L}} Q_{l}(t) R_{l}(t)
$$

(ties are broken arbitrarily).

"Good" tie-breaking rule: Assume that the tie-breaking rule is applied to pick a short-lived flow every time slot (but the flow is served only if $\alpha \sum_{i \in \mathcal{I}(t)}\left[\frac{Q_{i}(t)}{\tilde{R}_{i}^{\max (t)}}\right]>$ $\left.\max _{l \in \mathcal{L}} Q_{l}(t) R_{l}(t)\right)$. We define $\mathcal{E}_{\text {miss }}(t)$ to be the event that the tie-breaking rule selects a short-lived flow with $\tilde{R}_{i}^{\max }(t) \neq$ $R_{i}^{\max }$. A tie-breaking rule is said to be good if the following condition holds: Consider the WSL with the given tie-breaking rule and learning period $D$. Given any $\epsilon_{\text {miss }}>0$, there exist $N_{\epsilon_{m i s s}}$ and $D_{\epsilon_{m i s s}}$ such that

$$
\operatorname{Pr}\left(\mathcal{E}_{\text {miss }}(t)\right) \leq \epsilon_{\text {miss }}
$$

if $D \geq D_{\epsilon_{m i s s}}$ and $W_{s}(t-D) \geq N_{\epsilon_{m i s s}}$.

Remark 1: While all WSL scheduling algorithms with good tie-breaking rules are throughput optimal, their performances in terms of other metrics could be different depending upon the tie-breaking rules. We consider two tie-breaking rules in this paper:

- Uniform Tie-breaking: Among all short-lived flows satisfying $R_{i}(t)=\tilde{R}_{i}^{\max }(t)$ or $R_{i}(t) \geq Q_{i}(t)$, the basestation uniformly and randomly selects one to serve.
- Oldest-first Tie-breaking: Let $\beta_{i}$ denote the number of time slots a short-lived flow has been in the network. The base station keeps track $\tau_{i}=\min \left\{\bar{\tau}, \beta_{i}\right\}$ for every short-lived flow, where $\bar{\tau}$ is some fixed positive integer. Among all short-lived flows satisfying $R_{i}(t)=\tilde{R}_{i}^{\max }(t)$ or $R_{i}(t) \geq Q_{i}(t)$, the tie-breaking rule selects the one with the largest $\tau_{i}$, and the ties are broken uniformly and randomly 1

The "goodness" of these two tie-breaking rules are proved in Appendix $\mathrm{C}$ and $\mathrm{D}$, and the impact of the tie-breaking rules on performance is studied in Section VI using simulations.

Remark 2: The $\alpha$ in inequality (1) is a parameter balancing the performance of long-lived flows and short-lived flows. A large $\alpha$ will lead to a small number of short-lived flows but large queue-lengths of long-lived flows, and vice versa.

Remark 3: In Theorem 3, we will prove that WSL is throughput optimal when $D$ is sufficiently large. From purely through-optimality considerations, it is then natural to choose $D=\infty$. However, in practical systems, if we choose $D$ too large, such as $\infty$, then it is possible that a flow may stay in the system for a very long time if its best channel condition occurs extremely rarely. Thus, it is perhaps best to choose a finite $D$ to tradeoff between performance and throughput.

Remark 4: If all flows are short-lived, then the algorithm simplifies as follows: If at least one short-lived flow (say flow i) satisfies $R_{i}(t) \geq Q_{i}(t)$ or $R_{i}(t)=\tilde{R}_{i}^{\max }(t)$, then the base station uniformly and randomly selects such a flow for transmission (ties are broken arbitrarily); otherwise, the base station picks an arbitrary short-lived flow to serve. Simply stated, the algorithm serves one of the flows which can be completely transmitted or sees its best channel state, where the best channel state is an estimate based on past observations. If no such flow exists, any flow can be served. We do not separately prove the throughput optimality of this scenario since it is a special case of the scenario considered here. But it is useful to note that, in the case of short-lived flows only, the algorithm does not consider backlogs at all in making scheduling decisions.

We will prove that WSL (with any $\alpha>0$ ) is throughputoptimal in the following sections, i.e., the scheduling policy can support any set of traffic flows that are supportable by any other algorithm. In the next section, we first present the necessary conditions for the stability, which also define the network throughput region.

\section{NeCESSARY CONDITIONS FOR StABility}

In this section, we establish the necessary conditions for the stability of networks with flow-level dynamics. First, we introduce the following definitions and notations:

- We classify the short-lived flows into different classes. Class- $k$ is associated with random variables $\hat{R}_{k}$ and $\hat{F}_{k}{ }_{2}^{2}$

\footnotetext{
${ }^{1}$ We set a upper bound $\bar{\tau}$ on $\beta$ for technical reasons that facilitate the throughput-optimality proof. Since $\bar{\tau}$ can be arbitrarily large, we conjecture that this upper bound is only for analysis purpose, and not required in practical systems.

${ }^{2}$ We use ${ }^{\wedge}$ to indicate that the notation is associated with a class of shortlived flows instead of an individual short-lived flow.
} 
A short-lived flow $i$ belongs to class $k$ if $R_{i}(t)$ has the same distribution as $\hat{R}_{k}$ and the size of flow $i\left(f_{i}\right)$ has the same distribution as $\hat{F}_{k}$. We let $\Lambda_{k}(t)$ denote the number of class- $k$ flows joining the network at time $t$, where $\Lambda_{k}(t)$ are i.i.d. across time slots and $\mathbf{E}\left[\Lambda_{k}(t)\right]=\lambda_{k}$. Denote by $\mathcal{K}$ the set of distinct classes. We assume that $\mathcal{K}$ is finite, $|\mathcal{K}|=K$, and $\Lambda_{k}[t] \leq \lambda^{\max }$ for all $t$ and $k \in \mathcal{K}$.

- Let $\mathbf{c}$ denote an $L$-dimensional vector describing the state of the channels of the long-lived flows. In state $\mathbf{c}, R_{\mathbf{c}, l}$ is the service rate that long-lived flow $l$ can receive if it is scheduled. We denote by $\mathcal{C}$ the set of all possible states.

- Let $\mathbf{C}(t)$ denote the state of the long-lived flows at time $t$, and $\pi_{\mathbf{c}}$ denote the probability that $\mathbf{C}(t)$ is in state $\mathbf{c}$.

- Let $p_{\mathbf{c}, l}$ be the probability that the base station serves flow $l$ when the network is in state $\mathbf{c}$. Clearly, for any $\mathbf{c}$, we have

$$
\sum_{l \in \mathcal{L}} p_{\mathbf{c}, l} \leq 1
$$

Note that the sum could be less than 1 if the base station schedules a short-lived flow in this state.

- Let $\mu_{\mathbf{c}, s}$ be the probability that the base station serves a short-lived flow when the network is in state $\mathbf{c}$.

- Let $\Theta_{k, \beta}(t)$ denote the number of short-lived flows that belong to class- $k$ and have residual size $Q(t)=\beta$. Note that $\beta$ can only take on a finite number of values.

Theorem 1: Consider traffic parameters $\left\{x_{l}\right\}$ and $\left\{\lambda_{k}\right\}$, and suppose that there exists a scheduling policy guaranteeing

$$
\lim _{t \rightarrow \infty} \mathbf{E}\left[\sum_{l \in \mathcal{L}} Q_{l}(t)+\sum_{k \in \mathcal{K}} \sum_{\beta=1}^{F^{\max }} \Theta_{k, \beta}(t)\right]<\infty .
$$

Then there exist $p_{\mathbf{c}, l}$ and $\mu_{\mathbf{c}, s}$ such that the following inequalities hold:

$$
\begin{gathered}
\sum_{l \in \mathcal{L}} p_{\mathbf{c}, l} \leq 1 \quad \forall \mathbf{c} \in \mathcal{C} \\
x_{l} \leq \sum_{\mathbf{c} \in \mathcal{C}} \pi_{\mathbf{c}} p_{\mathbf{c}, l} R_{\mathbf{c}, l} \quad \forall l \in \mathcal{L} \\
\sum_{k \in \mathcal{K}} \lambda_{k} \mathbf{E}\left[\left[\frac{\hat{F}_{k}}{\hat{R}_{k}^{\max }}\right]\right] \leq \sum_{\mathbf{c} \in \mathcal{C}} \mu_{\mathbf{c}, s} \pi_{\mathbf{c}} . \\
\left(\sum_{l \in \mathcal{L}} p_{\mathbf{c}, l}\right)+\mu_{\mathbf{c}, s} \leq 1 \forall c \in \mathcal{C} .
\end{gathered}
$$

Proof: Note that $\sum_{c} \pi_{c} p_{c, l} R_{c, l}$ and $\sum_{\mathbf{c} \in \mathcal{C}} \mu_{\mathbf{c}, s} \pi_{\mathbf{c}}$ are the mean service rates allocated to long-lived flow $l$ and shortlived flows respectively, so inequality (3) and (4) indicate that the mean service rates should be no less than the mean arrival rates.

Next, we note that $\lambda_{k} \mathbf{E}\left[\left[\frac{\hat{F}_{k}}{\hat{R}_{k}^{\max }}\right]\right]$ is the expected number of time slots required to transmit short-lived flows belonging to class- $k$ assuming the flows are always served with rate $\hat{R}_{k}^{\max }$. Inequality (5) states that the overall time used to serve longlived and short-lived flows should be no more than the time available. The complete proof is based on the Strict Separation Theorem and is along the lines of a similar proof in [4].

\section{Throughrut Optimality OF WSL}

First, we provide some intuition into how one can derive the WSL algorithm from optimization decomposition considerations. Then, we will present our main throughput optimality results. Given traffic parameters $\left\{x_{l}\right\}$ and $\left\{\lambda_{k}\right\}$, the necessary conditions for the supportability of the traffic is equivalent to the feasibility of the following constraints:

$$
\begin{array}{cc}
x_{l} \leq \sum_{\mathbf{c} \in \mathcal{C}} \pi_{\mathbf{c}} p_{\mathbf{c}, l} R_{\mathbf{c}, l} & \forall l \\
\sum_{k \in \mathcal{K}} \lambda_{k} \mathbf{E}\left[\left[\frac{\hat{F}_{k}}{\hat{R}_{k}^{\max }}\right]\right] \leq \sum_{\mathbf{c} \in \mathcal{C}} \mu_{\mathbf{c}, s} \pi_{\mathbf{c}} & \\
\sum_{l \in \mathcal{L}} p_{\mathbf{c}, l}+\mu_{\mathbf{c}, s} \leq 1 & \forall \mathbf{c} .
\end{array}
$$

For convenience, we view the feasibility problem as an optimization problem with the objective $\max A$, where $A$ is some constant. While we have not explicitly stated that the $x$ 's and $\mu$ 's are non-negative, this is assumed throughout.

Partially augmenting the objective using Lagrange multipliers, we get

$$
\begin{array}{cc} 
& \max A-\sum_{l \in \mathcal{L}} q_{l}\left(x_{l}-\sum_{c} \pi_{\mathbf{c}} p_{\mathbf{c}, l} R_{\mathbf{c}, l}\right)- \\
& q_{s}\left(\sum_{k \in \mathcal{K}} \lambda_{k} \mathbf{E}\left[\left[\frac{\hat{F}_{k}}{\hat{R}_{k}^{\max }}\right]\right]-\sum_{\mathbf{c} \in \mathcal{C}} \mu_{\mathbf{c}, s} \pi_{\mathbf{c}}\right) \\
\text { s.t. } & \sum_{l \in \mathcal{L}} p_{\mathbf{c}, l}+\mu_{\mathbf{c}, s} \leq 1 \forall \mathbf{c} .
\end{array}
$$

For the moment, let us assume Lagrange multipliers $q_{l}$ and $q_{s}$ are given. Then the maximization problem above can be decomposed into a collection of optimization problems, one for each $\mathbf{c}$ :

$$
\begin{array}{lc} 
& \max _{\mathbf{p}_{\mathbf{c}}, \mu_{\mathbf{c}}, s} \sum_{l \in \mathcal{L}} q_{l} R_{\mathbf{c}, l} p_{\mathbf{c}, l}+q_{s} \mu_{\mathbf{c}, s} \\
\text { s.t. } \quad & \sum_{l \in \mathcal{L}} p_{\mathbf{c}, l}+\mu_{\mathbf{c}, s} \leq 1 .
\end{array}
$$

It is easy to verify that one optimal solution to the optimization problem above is:

- if $q_{s}>\max _{l \in \mathcal{L}} q_{l} R_{\mathbf{c}, l}$, then $\mu_{\mathbf{c}, s}=1$ and $p_{\mathbf{c}, l}=0(\forall l)$;

- otherwise, $\mu_{\mathbf{c}, s}=0$, and $p_{\mathbf{c}, l^{*}}=1$ for some $l^{*} \in$ $\arg \max q_{l} R_{\mathbf{c}, l}$ and $p_{\mathbf{c}, l}=0$ for other $l$.

The complementary slackness conditions give

$$
q_{l}\left(x_{l}-\sum_{\mathbf{c} \in \mathcal{C}} \pi_{\mathbf{c}} p_{\mathbf{c}, l} R_{\mathbf{c}, l}\right)=0 .
$$

Since $x_{l}$ is the mean arrival rate of long-lived flow $l$ and $\sum_{\mathbf{c} \in \mathcal{C}} \pi_{\mathbf{c}} p_{\mathbf{c}, l} R_{\mathbf{c}, l}$ is the mean service rate, the condition on $q_{l}$ says that if the mean arrival rate is less than the mean service rate, $q_{l}$ is equal to zero. Along with the non-negativity condition on $q_{l}$, this suggests that perhaps $q_{l}$ behaves likes a queue with these arrival and service rates. Indeed, it turns out that the mean of the queue lengths are proportional to Lagrange multipliers (see the surveys in [8]-[10]). For long-lived flow $l$, we can treat the queue-length $Q_{l}(t)$ as a time-varying estimate of Lagrange multiplier $q_{l}$. Similarly $q_{s}$ can be associated with a queue whose arrival rate is $\sum_{k \in \mathcal{K}} \lambda_{k} \mathbf{E}\left[\left[\frac{\hat{F}_{k}}{\hat{R}_{k}^{\max }}\right]\right]$, which is the mean rate at which workload arrives where workload is measured by the number of slots needed to serve a short-lived flow if it is served when its channel condition is the best. The 
service rate is $\sum_{\mathbf{c} \in \mathcal{C}} \mu_{\mathbf{c}, s} \pi_{\mathbf{c}}$ which is the rate at which the workload can potentially decrease when a short-lived flow is picked for scheduling by the base station. Thus, the workload in the system can serve as a dynamic estimate of $q_{s}$.

Letting

$$
W_{s}(t)=\sum_{i \in \mathcal{I}(t)}\left\lceil\frac{Q_{i}(t)}{R_{i}^{\max }}\right\rceil
$$

and $\alpha W_{s}(t)(\alpha>0)$ be an estimate of $q_{s}$, the observations above suggest the following workload-based scheduling algorithm if $R_{i}^{\max }$ are known.

Workload-based Scheduling (WS): During each time slot, the base station checks the following inequality:

$$
\alpha W_{s}(t)>\max _{l \in \mathcal{L}} Q_{l}(t) R_{l}(t) .
$$

- If inequality (7) holds, then the base station serves a shortlived flow as follows: if at least one short-lived flow (say flow $i$ ) satisfies $R_{i}(t) \geq Q_{i}(t)$ or $R_{i}(t)=R_{i}^{\max }$, then such a flow is selected for transmission (ties are broken arbitrarily); otherwise, the base station picks an arbitrary short-lived flow to serve.

- If inequality (7) does not hold, then the base station serves a long-lived flow $l^{*}$ such that $l^{*} \in \arg \max _{l \in \mathcal{L}} Q_{l}(t) R_{l}(t)$ (ties are broken arbitrarily).

- The factor $\alpha$ can be obtained from the optimization formulation by multiplying constraint 6 by $\alpha$ on both sides

However, this algorithm which was directly derived from dual decomposition considerations is not implementable since $R_{i}^{\max }$ 's are unknown. So WSL uses $\tilde{R}_{i}^{\max }(t)$ to approximate $R_{i}^{\max }$. Note that an inaccurate estimate of $R_{i}^{\max }$ not only affects the base station's decision on whether $R_{i}(t)=R_{i}^{\max }$, but also on its computation of $\left[\frac{Q_{i}(t)}{R_{i}^{\max }}\right]$. However, it is not difficult to see that the error in the estimate of the total workload is a small fraction of the total workload when the total workload is large: when the workload is very large, the total number of short-lived flows is large since their file sizes are bounded. Since the arrival rate of short-lived flows is also bounded, this further implies that a large number of shortlived flows must have arrived a long time ago which means that with high probability, their estimate of their best channel condition must be correct.

Next we will prove that both WS and WSL can stabilize any traffic $X_{l}(t)$ and $\Lambda_{k}(t)$ such that $(1+\epsilon) X_{l}(t)$ and $(1+\epsilon) \Lambda_{k}(t)$ are supportable, i.e., satisfying the conditions presented in Theorem 1 Even though WS is not practical, we study it first since the proof of its throughput optimality is easier and provides insight into the proof of throughput-optimality of WSL.

Let

$$
\mathbf{M}(t)=\left(\left\{Q_{l}(t)\right\}_{l \in \mathcal{L}},\left\{\Theta_{k, \beta}(t)\right\}_{k \in \mathcal{K}, 1 \leq \beta \leq F^{\max }}\right) .
$$

Since the base station makes decisions on $\mathbf{M}(t)$ and $\mathbf{R}(t)=$ $\left\{\left\{R_{i}(t)\right\}_{i \in \mathcal{I}(t)},\left\{R_{l}(t)\right\}_{l \in \mathcal{L}}\right\}$ under WS. It is easy to verify that $\mathbf{M}(t)$ is a finite-dimensional Markov chain under WS.
Assume that $\Lambda_{k}$ and $\hat{F}_{k}$ are such that the Markov chain $\mathbf{M}$ is irreducible and aperiodic.

Theorem 2: Given any traffic $X_{l}(t)$ and $\Lambda_{k}(t)$ such that $(1+\epsilon) X_{l}(t)$ and $(1+\epsilon) \Lambda_{k}(t)$ are supportable, the Markov chain $\mathbf{M}(t)$ is positive-recurrent under WS, which implies that $\lim _{t \rightarrow \infty} \mathbf{E}[\mathbf{M}(t)]<\infty$ under WS.

Proof: We consider the following Lyapunov function:

$$
V(t)=\alpha\left(W_{s}(t)\right)^{2}+\sum_{l \in \mathcal{L}}\left(Q_{l}(t)\right)^{2}
$$

and prove that

$$
\mathbf{E}[V(t+1)-V(t) \mid \mathbf{M}(t)] \leq U_{d} 1_{\mathbf{M}(t) \in \Upsilon}-U 1_{\mathbf{M}(t) \notin \Upsilon},
$$

for some $U_{d}>0, U>0$, and a finite set $\Upsilon$. Positive recurrence of $\mathbf{M}$ then follows from Foster's Criterion for Markov chains [11]. The detailed proof is presented in Appendix A.

We next study WSL, where $R_{i}^{\max }$ is estimated from the history. We define $\Theta_{k, \beta, r}(t)$ to be the number of short-lived flows that belong to class- $k$, have a residual size of $\beta$, and have $\tilde{R}_{i}^{\max }(t)=r$. Furthermore, we define

$$
\tilde{\mathbf{M}}(n)=\left(\left\{Q_{l}(t)\right\}_{l \in \mathcal{L}},\left\{\Theta_{k, \beta, r}(t)\right\}_{\substack{1 \leq \beta \leq F^{\max } \\ 1 \leq r \leq \hat{R}_{k}^{\max }}}\right)_{(n-1) T+1 \leq t \leq n T}
$$

from some $T \geq D$. It is easy to see that $\tilde{\mathbf{M}}(n)$ is a finitedimensional Markov chain under WSL 3

Theorem 3: Consider traffic $X_{l}(t)$ and $\Lambda_{k}(t)$ such that $(1+$ $\epsilon) X_{l}(t)$ and $(1+\epsilon) \Lambda_{k}(t)$ are supportable. Given WSL with a good tie-breaking rule, there exists $D_{\epsilon}$ such that the Markov chain $\tilde{\mathbf{M}}(n)$ is positive-recurrent under the WSL with learning period $D \geq D_{\epsilon}$ and the given tie-breaking rule.

Proof: The proof of this theorem is built upon the following two facts:

- When the number of short-lived flows is large, the majority of short-lived flows must have been in a network for a long time and have obtained the correct estimate of the best channel condition, which implies that

$$
\sum_{i \in \mathcal{I}(t)}\left\lceil\frac{Q_{i}(t)}{R_{i}^{\max }}\right\rceil \approx \sum_{i \in \mathcal{I}(t)}\left\lceil\frac{Q_{i}(t)}{\tilde{R}_{i}^{\max }(t)}\right\rceil .
$$

- When the number of short-lived flows is large, the shortlived flow selected by the base station (say flow $i$ ) has a high probability to satisfy $R_{i}(t)=R_{i}^{\max }$ or $R_{i}(t) \geq Q_{i}(t)$.

From these two facts, we can prove that with a high probability, the scheduling decisions of WSL are the same as those of WS, which leads to the throughput optimality of WSL. The detailed proof is presented in Appendix B.

\footnotetext{
${ }^{3}$ This Markov chain is well-defined under the uniform tie-breaking rule. For other good tie-breaking rules, we may need to first slightly change the definition of $\tilde{M}(n)$ to include the information required for tie-breaking, and then use the analysis in Appendix B to prove the positive recurrence.
} 


\section{Simulations}

In this section, we use simulations to evaluate the performance of different variants of WSL and compare it to other scheduling policies. There are three types of flows used in the simulations:

- S-flow: An S-flow has a finite size, generated from a truncated exponential distribution with mean value 30 and maximum value 150 . Non-integer values are rounded to integers.

- M-flow: An M-flow keeps injecting bits into the network for 10,000 time slots and leaves the network. The number of bits generated at each time slot follows a Poisson distribution with mean value 1 .

- L-flow: An L-flow keeps injecting bits into the network and never leaves the network. The number of bits generated at each time slot follows a Poisson distribution with mean value 1 .

Here S-flows represent short-lived flows that have finite sizes and whose bits arrive all at once; L-flows represent longlived flows that continuously inject bits and never leave the network; and M-flows represent flows of finite size but whose arrival rate is controlled at their sources so that they od not arrive instantaneously into the network. Our simulation will demonstrate the importance of modeling very large, but finitesized flows as long-lived flows.

We assume that the channel between each user and the base station is distributed according to one of the following three distributions:

- G-link: A G-link has five possible link rates $\{10,20,30,40,50\}$, and each of the states happens with probability $20 \%$.

- P-link: A P-link has five possible link rates $\{5,10,15,20,25\}$, and each of the states happens with probability $20 \%$.

- R-link: An R-link has six possible link rates $\{10,20,30,40,100\}$, and the probabilities associated with these link states are $\{0.5,0.2,0.2,0.09,0.01\}$.

The G, P and R stand for Good, Poor and Rare, respectively. We include these three different distributions to model the SNR variations among the users, where G-links represent links with high SNR (e.g., those users close to the base station), Plinks represent links with low SNR (e.g., those users far away from the base station), and R-links represent links whose best state happens rarely. The R-links will be used to study the impact of learning period $D$ on the network performance.

We name the WSL with the uniform tie-breaking rule WSLU, and the WSL with the oldest-first tie-breaking rule WSLO. In the following simulations, we will first demonstrate that the WSLU performs significantly better than previously suggested algorithms, and then show that the performance can be further improved by choosing a good tie-breaking policy (e.g., WSLO). We set $\alpha$ to be 50 in all the following simulations.
Simulation I: Short-lived Flow or Long-lived Flow?

We first use the simulation to demonstrate the importance of considering a flow with a large number of packets as being long-lived. We consider a network consisting of multiple Sflows and three M-flows, where the arrival of S-flows follows a truncated Poisson process with maximum value 100 and mean value $\lambda$. All the links are assumed to be G-links. We evaluate the following two schemes:

- Scheme-1: Both S-flows and M-flows are considered to be short-lived flows.

- Scheme-2: An M-flow is considered to be long-lived before its last packet arrives, and to be short-lived after that.

The performance of these two schemes are shown in Figure 1 where WS with Uniform Tie-breaking Rule is used as the scheduling algorithm. We can see that the performances are substantially different (note that the network is stable under both schemes). The number of queued bits of $\mathrm{M}$ flows under Scheme-1 is larger than that under Scheme-2 by two orders of magnitude. This is because even an M-flow contains a huge number of bits (10,000 on average), it can be served only when the link rate is 50 under Scheme-1. This simulation suggests that when the performance we are interested is at a small scale (e.g. acceptable queue-length being $\leq 100$ ) compared with the size of the flow (e.g., $10^{4}$ in this simulation), the flow should be viewed a long-lived flow for performance purpose.

\section{Simulation II: The Impact of Learning Period D}

In this simulation, we investigate the impact of $D$ on the performance of WSLU. Recall that it is nature to choose $D=\infty$ for purely throughput-optimality considerations, but the disadvantage is that a flow may stay in the network for a very long time if the best link state occurs very rarely. We consider a network consisting of S-flows, which arrive according to a truncated Poisson process with maximum value 100 and mean $\lambda$, and three L-flows. All links are assumed to be R-links. Figure 2 depicts the mean and standard deviation of the file-transfer delays with $D=16$ and $D=\infty$. As we expected, the standard deviation under WSLU with $D=\infty$ is significantly larger than that under WSLU with $D=16$. This occurs because the best link rate 100 occurs with a probability 0.01 . This simulation confirms that in practical systems, we may want to choose a finite $D$ to get desired performance. In the following simulations, we choose $D=16$.

In the following simulations, we choose $D=16$. In the introduction, we have pointed out that the MaxWeight is not throughput optimal under flow-level dynamics because the backlog of a short-lived queue does not build up even when it has not being served for a while. To overcome this, one could try to use the delay of the head-of-line packet, instead of queue-length, as the weight because the head-of-line delay will keep increasing if no service is received. In the case of long- 

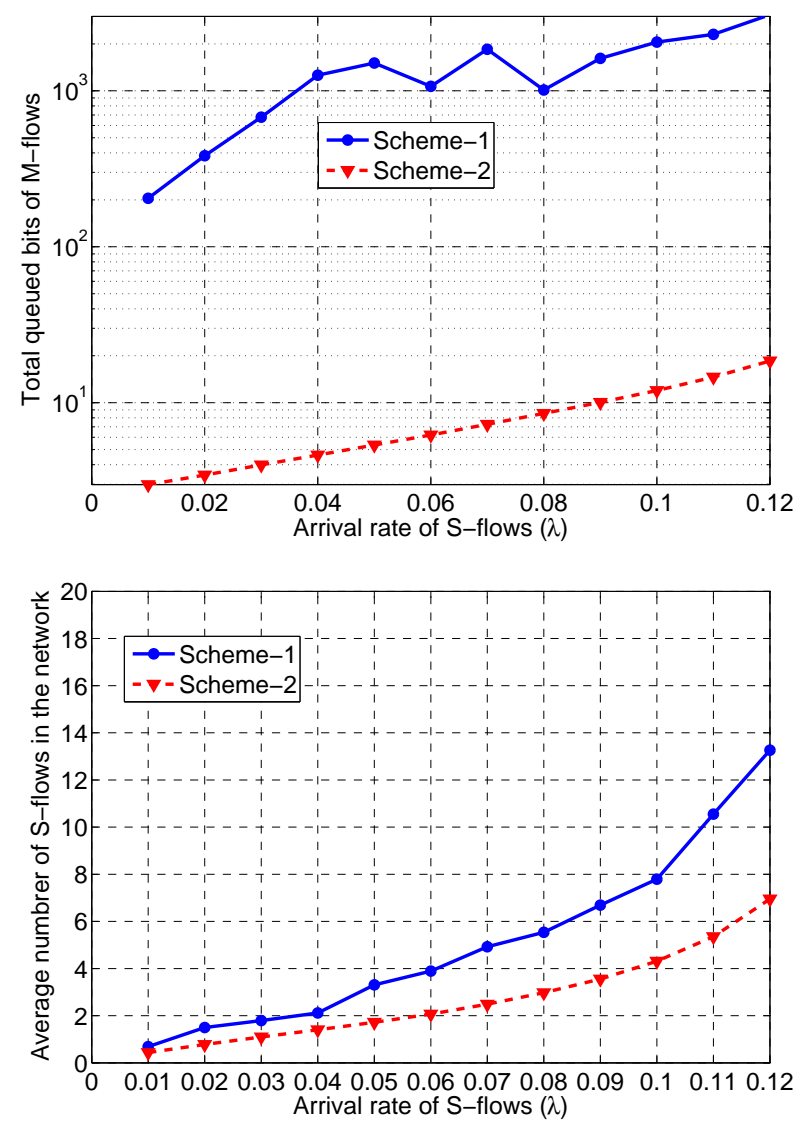

Fig. 1. Scheme-1 treats M-flows as short-lived flows, and Scheme-2 treats M-flows as long-lived flows.

lived flows only, this algorithm is known to be throughputoptimal [4]. We will show that this Delay-based scheduling does not solve the instability problem when there are shortlived flows.

Delay-based Scheduling: At each time slot, the base station selects a flow $i$ such that $i \in \arg \max _{i} D_{i}(t) R_{i}(t)$, where $D_{i}(t)$ is the delay experienced so far by the head-of-line packet of flow $i$.

\section{Simulation III: Performance comparison of various algorithms}

We first consider the case where all flows are S-flows, which arrive according to a truncated Poisson process with maximum value 100 and mean $\lambda$. An S-flow is assigned with a G-link or a P-link equally likely.

Figure 3 shows the average file-transfer delay and average number of S-flows under different values of $\lambda$. We can see that WSLU performs significantly better than the MaxWeight and Delay-based algorithms. Specifically, under MaxWeight and Delay-based algorithms, both the number of S-flows and file-transfer delay explode when $\lambda \geq 0.102$. WSLU, on the other hand, performs well even when $\lambda=0.12$.

Next, we consider the same scenario with three L-flows in the network. Two of the L-flows have G-links and one has a
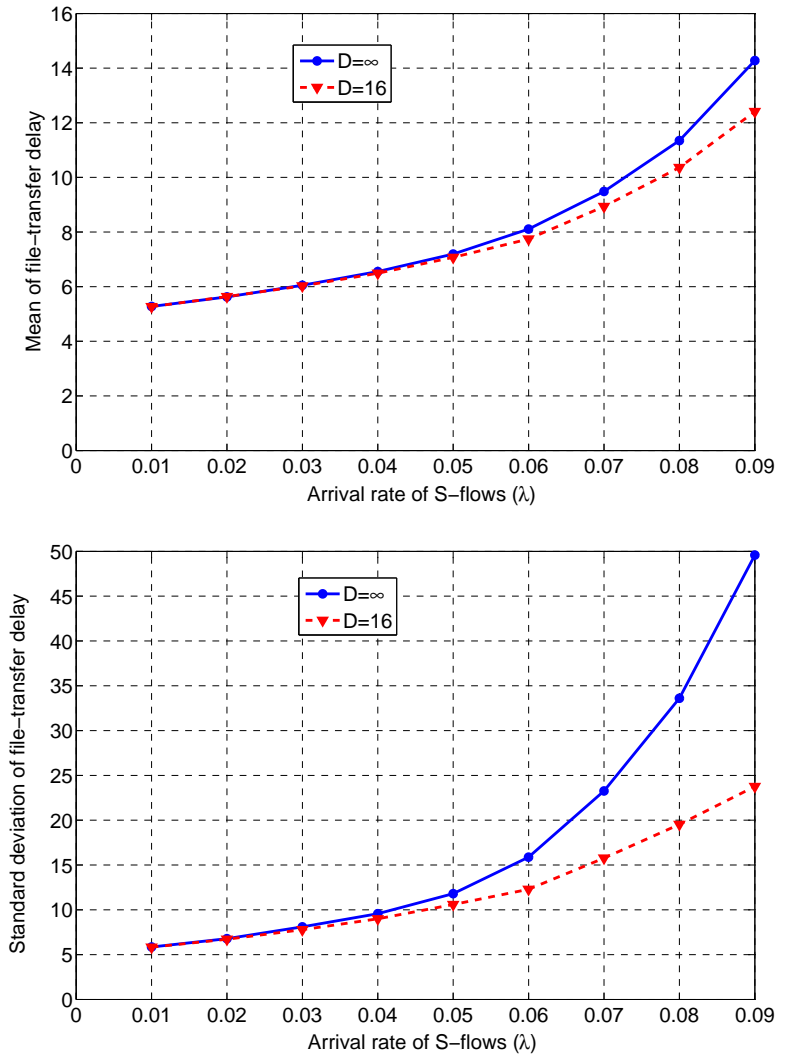

Fig. 2. The performance of WSLU with $D=16$ and $D=\infty$

P-link. Figure 4 shows the number of short-lived flows and file-transfer delay under different values of $\lambda$. We can see that the MaxWeight becomes unstable even when the arrival rate of $S$-flows is very small. This is because the MaxWeight stops serving S-flows when the backlogs of F-flows are large, so Sflows stay in the network forever. The delay-based scheduling performs better than the MaxWeight, but significantly worse than WSLU.

\section{Simulation IV: Blocking probability of various algorithms}

While our theory assumes that the number of flows in the network can be infinite, in reality, base stations limit the number of simultaneously active flows, and reject new flows when the number of existing flows above some threshold. In this simulation, we assume that the base station can support at most $20 \mathrm{~S}$-flows. A new S-flow will be blocked if $20 \mathrm{~S}$ flows are already in the network. In this setting, the number of flows in the network is finite, so we compute the blocking probability, i.e., the fraction of S-flows rejected by the base station.

We consider the case where no long-lived flow is in the network and the case where both short-lived and long-lived flows are present in the network. The flows and channels are selected as in Simulation III. The results are shown in Figure 5 and 6 We can see that the blocking probability under WSLU is substantially smaller than that under the MaxWeight 

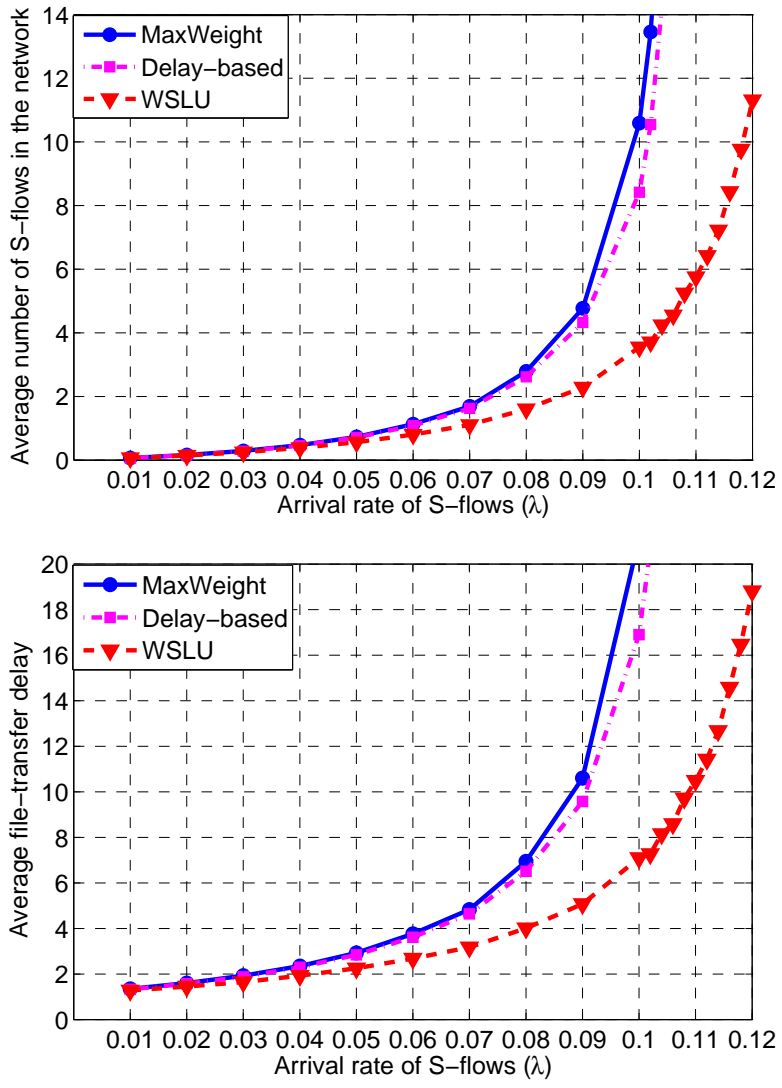

Fig. 3. The performance of the delay-based, MaxWeight, and WSLU algorithms in a network without long-lived flows

or delay-based scheduling. Thus, this simulation demonstrates that instability under the assumption when the number of flows is allowed to unbounded implies high blocking probabilities for the practical scenario when the base station limits the number of flows in the network.

\section{Simulation V: WSLU versus WSLO}

In this simulation, we study the impact of tie-breaking rules on performance. We compare the performance of the WSLU and WSLO. We first study the case where the base station does not limit the number of simultaneously active flows and there is no long-lived flow in the network. The simulation setting is the same as that in Simulation III. Figure 7 shows the average file-transfer delay and average number of S-flows under different values of $\lambda$. We can see that the WSLO reduces the file-transfer delay and number of S-flows by nearly $75 \%$ when $\lambda=0.13$, which indicates the importance of selecting a good tie-breaking rule for improving the network performance.

Next, we study the case where the base station does not limit the number of simultaneously active flows and there are three L-flows in the network. Figure 8 shows the number of short-lived flows and file-transfer delay under different values of $\lambda$. We can see again that the WSLO algorithm has a much better performance than the WSLU, especially when $\lambda$ is large.
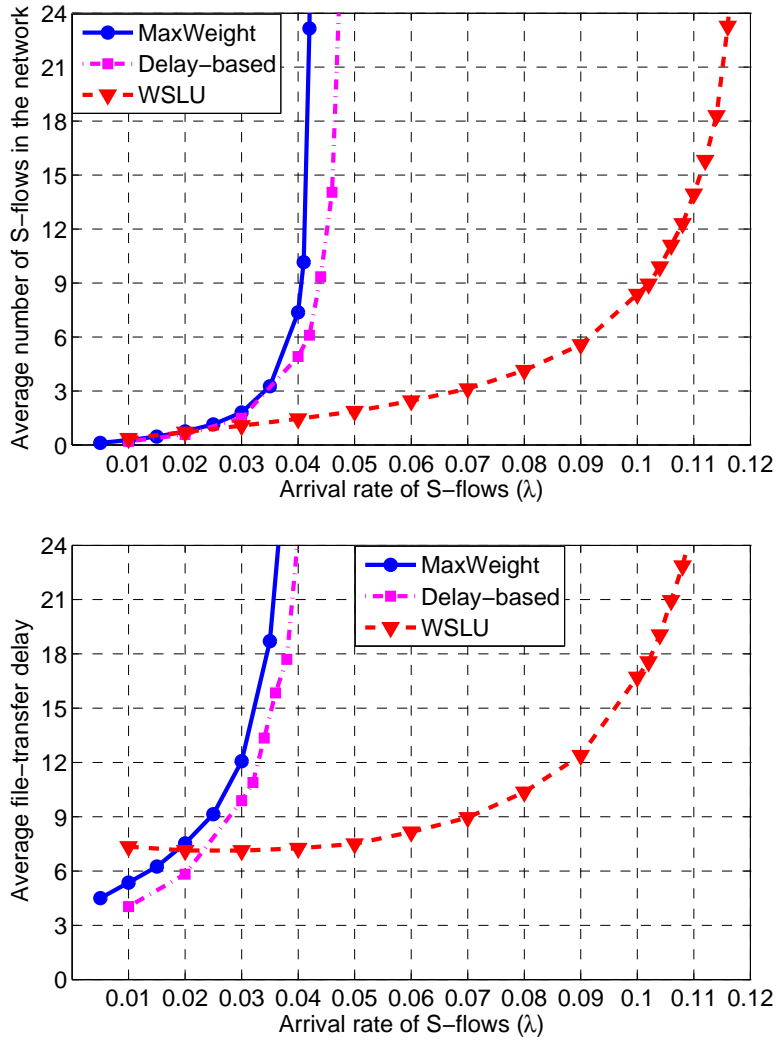

Fig. 4. The performance of the delay-based, MaxWeight, and WSLU algorithms in a network with both S-flows and L-flows

Finally we consider the situation in which the base station can support at most 20 S-flows. A new S-flow will be blocked if $20 \mathrm{~S}$-flows are already in the network. The simulation setting is the same as that in Simulation IV. We calculate the blocking probabilities, and the results are shown in Figure 9 and 10 We can see that the blocking probability under the WSLO is much smaller than that under the WSLU policy when $\lambda$ is large.

\section{CONCLUSIONS}

In this paper, we studied multiuser scheduling in networks with flow-level dynamics. We first obtained necessary conditions for flow-level stability of networks with both long-lived flows and short-lived flows. Then based on an optimization framework, we proposed the workload-based scheduling with learning that is throughput-optimal under flow-level dynamics and requires no prior knowledge about channels and traffic. In the simulations, we evaluated the performance of the proposed scheduling algorithms, and demonstrated that the proposed algorithm performs significantly better than the MaxWeight scheduling in various settings.

\section{ACKNOWLEDGMENTS}

Research supported by NSF Grants 07-21286 and 08-31756, ARO MURI Subcontracts, and the DTRA grants HDTRA1-081-0016 and HDTRA1-09-1-0055. 


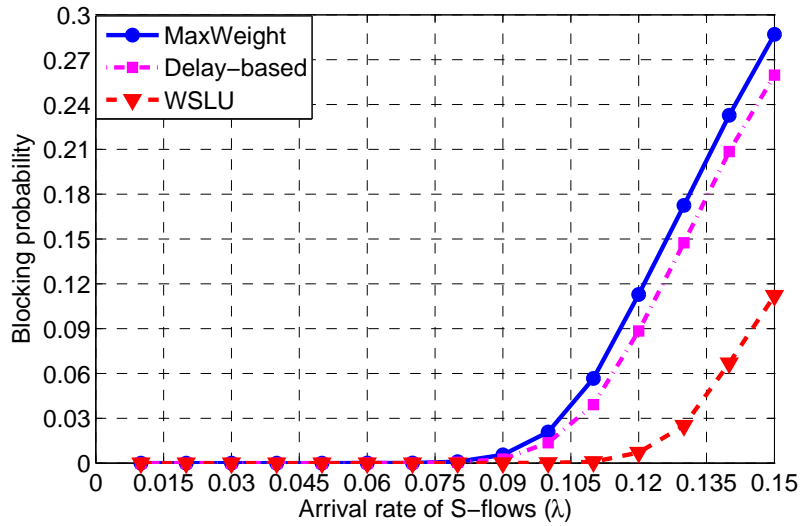

Fig. 5. The blocking probabilities of the delay-based, MaxWeight, and WSLU in a network without L-flow

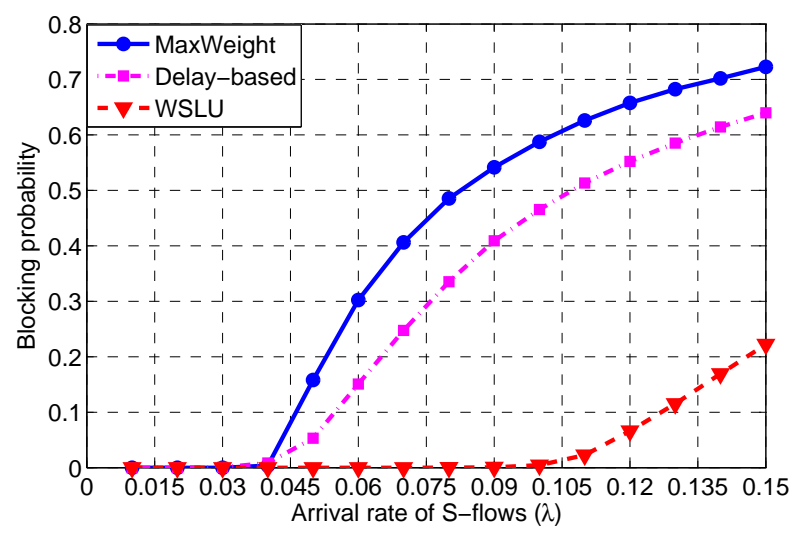

Fig. 6. The blocking probabilities of the delay-based, MaxWeight, and WSLU in a network with L-flow

\section{Appendix A: Proof of Theorem 2}

Recall that

$$
W_{s}(t)=\sum_{i \in \mathcal{I}(t)}\left\lceil\frac{Q_{i}(t)}{R_{i}^{\max }}\right\rceil
$$

We define

$$
A_{s}(t)=\sum_{k \in \mathcal{K}} \sum_{i \in \Lambda_{k}(t)}\left\lceil\frac{f_{i}}{\hat{R}_{k}^{\max }}\right\rceil,
$$

which is the amount of new workload (from short-lived flows) injected in the network at time $t$, and $\mu_{s}(t)$ to be the decrease of the workload at time $t$, i.e., $\mu_{s}(t)=1$ if the workload of short-lived flows is reduced by one and $\mu_{s}(t)=0$ otherwise. Based on the notations above, the evolution of short-lived flows can be described as:

$$
W_{s}(t+1)=W_{s}(t)+A_{s}(t)-\mu_{s}(t) .
$$

Further, the evolution of $Q_{l}(t)$ can be described as

$$
Q_{l}(t+1)=Q_{l}(t)+X_{l}(t)-\mu_{l}(t)+u_{l}(t),
$$

where $\mu_{l}(t)$ is the decrease of $Q_{l}(t)$ due to the service longlived flow $l$ receives at time $t$, and $u_{l}(t)$ is the unused service due to the lack of data in the queue.
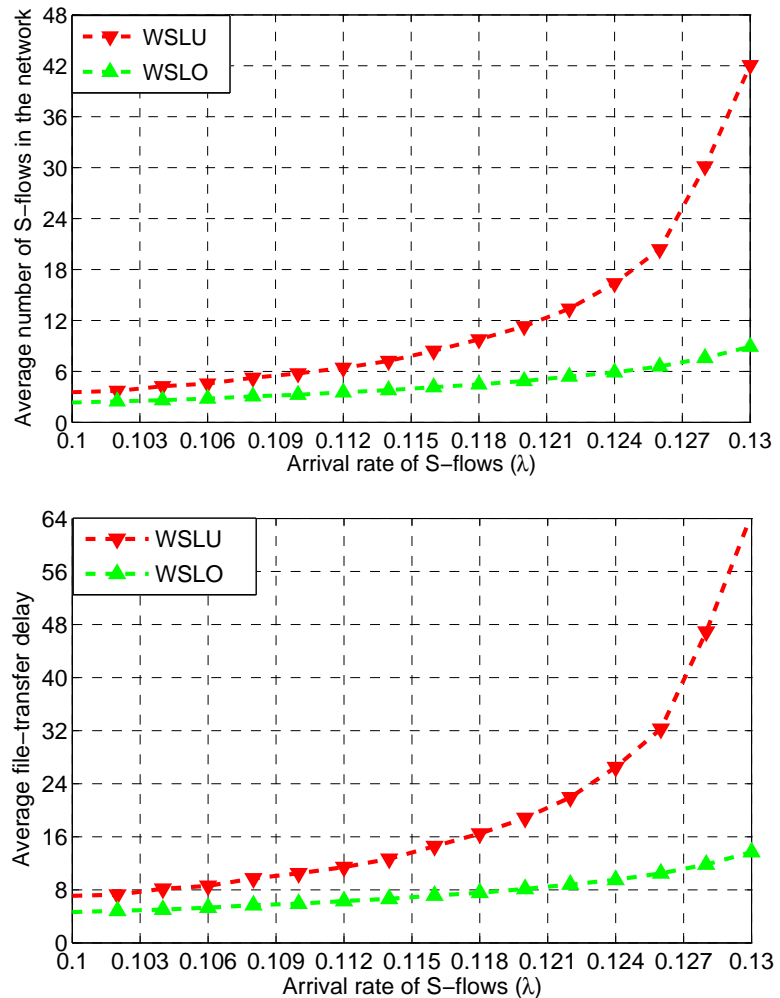

Fig. 7. The performance of the WSLU and WSLO algorithms in a network without L-flows

We consider the following Lyapunov function

$$
V(t)=\alpha\left(W_{s}(t)\right)^{2}+\sum_{l \in \mathcal{L}}\left(Q_{l}(t)\right)^{2}
$$

We will prove that the drift of the Lyapunov function satisfies

$$
\mathbf{E}[V(t+1)-V(t) \mid \mathbf{M}(t)] \leq U_{d} 1_{\mathbf{M}(t) \in \Upsilon}-U 1_{\mathbf{M}(t) \notin \Upsilon}
$$

for some $U_{d}>0, U>0$, and a finite set $\Upsilon$ (the values of these parameters will be defined in the following analysis). Positive recurrence of $\mathbf{M}$ then follows from Foster's Criterion for Markov chains [11].

First, since the number of arrivals, the sizes of short-lived flows and channel rates are all bounded, it can be verified that 

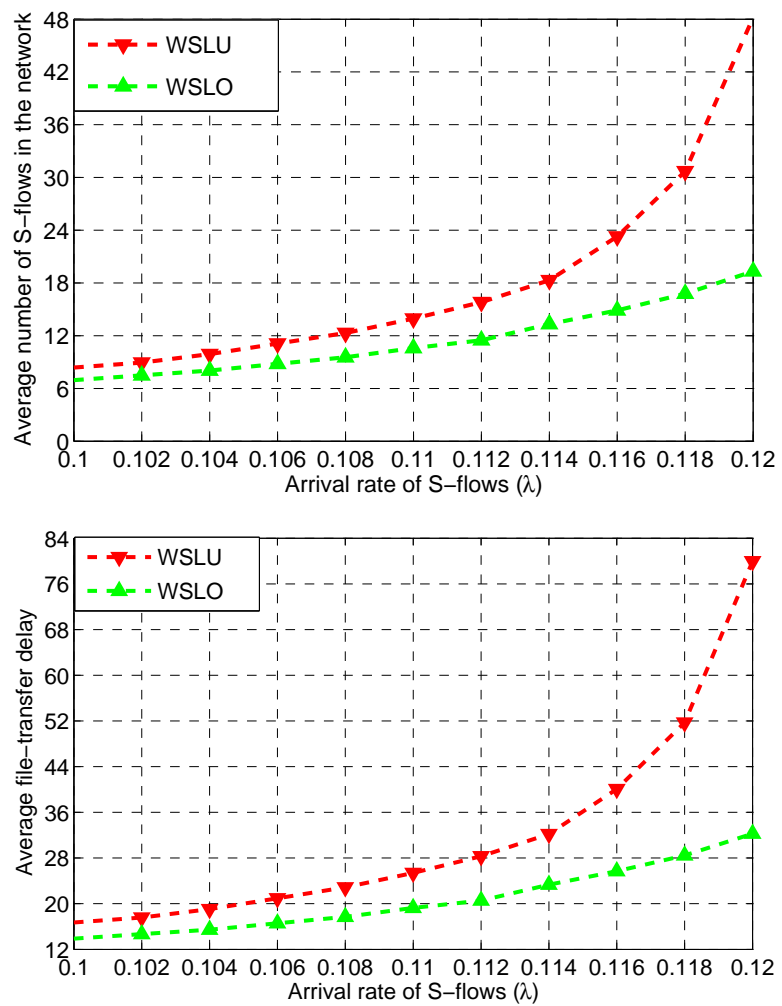

Fig. 8. The performance of the WSLU and WSLO algorithms in a network with both S-flows and L-flows

there exists $U$, independent of $\mathbf{M}(t)$, such that

$$
\begin{gathered}
\quad \mathbf{E}[V(t+1)-V(t) \mid \mathbf{M}(t)] \\
=\mathbf{E}\left[\alpha\left(W_{s}(t+1)\right)^{2}-\alpha\left(W_{s}(t)\right)^{2}+\right. \\
\left.\quad \sum_{l \in \mathcal{L}}\left(Q_{l}(t+1)\right)^{2}-\sum_{l \in \mathcal{L}}\left(Q_{l}(t)\right)^{2} \mid \mathbf{M}(t)\right] \\
\leq U+2 \alpha W_{s}(t) \mathbf{E}\left[A_{s}(t)-\mu_{s}(t) \mid \mathbf{M}(t)\right]+ \\
\quad 2 \sum_{l \in \mathcal{L}} Q_{l}(t) \mathbf{E}\left[X_{l}(t)-\mu_{l}(t) \mid \mathbf{M}(t)\right] \\
\leq U+2 \alpha W_{s}(t)\left(\left(\sum_{k \in \mathcal{K}} \lambda_{k} \mathbf{E}\left[\left|\frac{\hat{F}_{k}}{\hat{R}_{k}^{\max }}\right|\right]\right)\right. \\
+2 \sum_{l \in \mathcal{L}} Q_{l}(t)\left(x_{l}-\mathbf{E}\left[\mu_{s}(t) \mid \mathbf{M}(t)\right]\right)
\end{gathered}
$$

Recall that we assume that $(1+\epsilon) \mathbf{E}\left[X_{l}\right]$ and $(1+\epsilon) \mathbf{E}\left[\Lambda_{k}\right]$ satisfy the supportability conditions of Theorem 1 . By adding and subtracting corresponding $p_{\mathbf{c}, l} R_{\mathbf{c}, l}$ and $\mu_{\mathbf{c}, s}$, we obtain

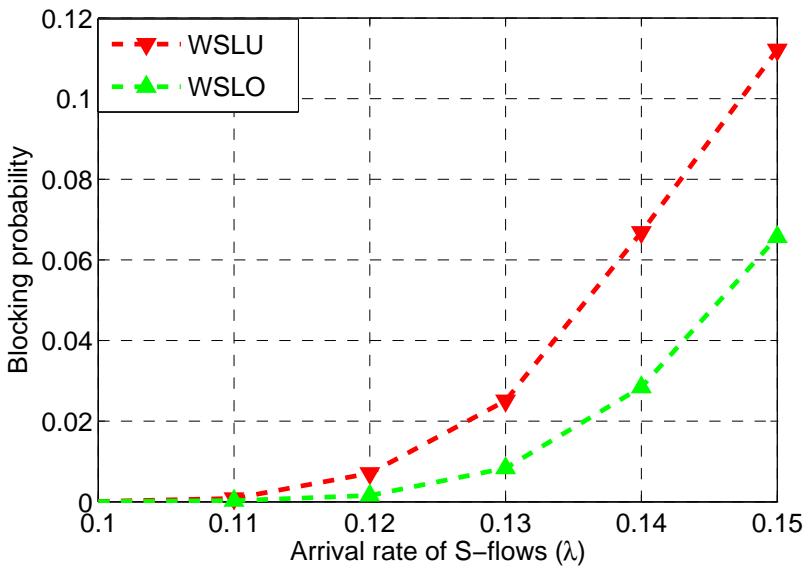

Fig. 9. The blocking probabilities of the WSLU and WSLO in a network without L-flows

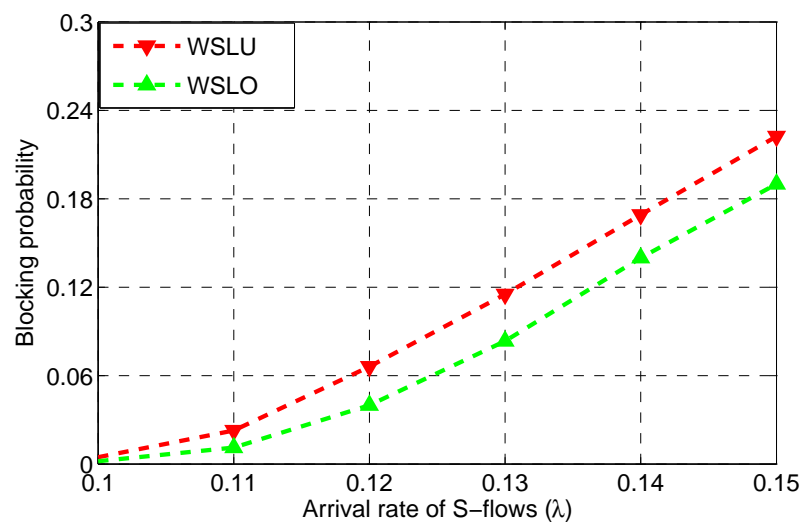

Fig. 10. The blocking probabilities of the WSLU and WSLO in a network with L-flows

that

$$
\begin{aligned}
& \mathbf{E}[V(t+1)-V(t) \mid \mathbf{M}(t)]-U \\
\leq \quad & 2 \alpha W_{s}(t) \mathbf{E}\left[\mathbf{E}\left[\mu_{\mathbf{c}, s}-\mu_{s}(t) \mid \mathbf{C}(t)=\mathbf{c}\right] \mid \mathbf{M}(t)\right] \\
& +2 \sum_{l \in \mathcal{L}} Q_{l}(t) \mathbf{E}\left[\mathbf{E}\left[p_{\mathbf{c}, l} R_{\mathbf{c}, l}-\mu_{l}(t) \mid \mathbf{C}(t)=\mathbf{c}\right] \mid \mathbf{M}(t)\right] \\
& -2 \epsilon \alpha W_{s}(t) \bar{\lambda}-2 \epsilon \sum_{l \in \mathcal{L}} Q_{l}(t) x_{l},
\end{aligned}
$$

where

$$
\bar{\lambda}=\left(\sum_{k \in \mathcal{K}} \lambda_{k} \mathbf{E}\left[\left\lceil\frac{\hat{F}_{k}}{\hat{R}_{k}^{\max }}\right\rceil\right]\right) .
$$

Next we assume $\mathbf{C}(t)=\mathbf{c}$ and analyze the following quantity

$$
\alpha W_{s}(t)\left(\mu_{\mathbf{c}, s}-\mu_{s}(t)\right)+\sum_{l \in \mathcal{L}} Q_{l}(t)\left(p_{\mathbf{c}, l} R_{\mathbf{c}, l}-\mu_{l}(t)\right) .
$$

We have the following facts:

- Fact 1: Assume that there exists a short-lived flow $i$ such that $R_{i}(t)=R_{i}^{\max }$ or $R_{i}(t) \geq Q_{i}(t)$. If a short-lived flow is selected to be served, then the workload of the 
selected flow is reduced by one and $\mu_{s}(t)=1$. If longlived flow $l$ is selected, the rate flow $l$ receives is $R_{\mathbf{c}, l}$. Thus, we have that

$$
\begin{aligned}
& \alpha W_{s}(t) \mu_{s}(t)+\sum_{l \in \mathcal{L}} Q_{l}(t) \mu_{l}(t) \\
= & \max \left\{\alpha W_{s}(t), \max _{l} Q_{l}(t) R_{\mathbf{c}, l}\right\} \\
\geq & \alpha W_{s}(t) \mu_{\mathbf{c}, s}+\sum_{l \in \mathcal{L}} Q_{l}(t) p_{\mathbf{c}, l} R_{\mathbf{c}, l},
\end{aligned}
$$

where the last inequality holds because $\sum_{l} p_{\mathbf{c}, l}+\mu_{\mathbf{c}, s} \leq$ 1. Therefore, we have

$$
\text { (10) } \leq 0
$$

in this case.

- Fact 2: Assume that there does not exist a short-lived flow $i$ such that $R_{i}(t)=R_{i}^{\max }$ or $R_{i}(t) \geq Q_{i}(t)$. In this case, we have

$$
\begin{aligned}
\text { (10) } & \leq \alpha W_{s}(t)+\max _{l \in \mathcal{L}} Q_{l}(t) R_{\mathbf{c}, l} \\
& \leq \alpha W_{s}(t)+R^{\max } \max _{l \in \mathcal{L}} Q_{l}(t) .
\end{aligned}
$$

Now we define a set $\Upsilon$ such that

$$
\Upsilon=\left\{\mathbf{M}: W_{s} \leq U_{W} \text { and } Q_{l} \leq U_{Q} \forall l\right\},
$$

where $U_{W}$ is a positive integer satisfying that

$$
\begin{aligned}
\left(1-p_{s}^{\max }\right)^{\frac{U_{W}}{F^{\operatorname{mIax}}}} \leq \frac{\epsilon}{2} \min \left\{\bar{\lambda}, \frac{\min _{l \in \mathcal{L}} x_{l}}{R^{\max }}\right\} \triangleq \epsilon_{1} \\
U_{W} \geq \frac{2 U}{\epsilon \alpha \lambda},
\end{aligned}
$$

and $U_{Q}$ is a positive integer satisfying

$$
U_{Q} \geq \frac{2 \alpha U_{W}+U}{\epsilon \min _{l \in \mathcal{L}} x_{l}} .
$$

We next compute the drift of the Lyapunov function according to the value of $\mathbf{M}(t)$.

- Case I: Assume $\mathbf{M}(t) \in \Upsilon$. According to the definition of $\Upsilon$, we have

$$
\mathbf{E}[V(t+1)-V(t) \mid \mathbf{M}(t)] \leq U+2 \alpha U_{W}+2 R^{\max } L U_{Q} .
$$

- Case II: Assume $W_{s}(t)>U_{W}$. Since the size of a shortlived flow is upper bounded by $F^{\max }, W_{s}(t)>U_{W}$ implies that at least $\frac{U_{W}}{F^{\max }}$ short-lived flows are in the network at time $t$. Define event $\mathcal{S}(t)$ such that $R_{i}(t)=$ $R_{i}^{\max }$ and $R_{i}(t) \geq Q_{i}(t)$ do not hold for any short-lived flow $i$.

Recall that

$$
\min _{i} \operatorname{Pr}\left(R_{i}(t)=R_{i}^{\max }\right) \geq p_{s}^{\max } .
$$

Given at least $\frac{U_{W}}{F^{\max }}$ short-lived flows are in the network, we have that

$$
\operatorname{Pr}\left(1_{\mathcal{S}(t)}=1\right) \leq\left(1-p_{s}^{\max }\right)^{\frac{U_{W}}{F^{\max }}} \leq \epsilon_{1}
$$

According to facts 1 and 2, (10) is positive only if $\mathcal{S}(t)$ occurs and the value of (10) is bounded by $\alpha W_{s}(t)+$
$R^{\max } \max _{l \in \mathcal{L}} Q_{l}(t)$. Therefore, we can conclude that in this case (Case II),

$$
\begin{aligned}
& \mathbf{E}[V(t+1)-V(t) \mid \mathbf{M}(t)] \\
\leq & U+2 \epsilon_{1}\left(\alpha W_{s}(t)+R^{\max } \max _{l \in \mathcal{L}} Q_{l}(t)\right) \\
& -2 \epsilon \alpha W_{s}(t) \bar{\lambda}-2 \epsilon \sum_{l \in \mathcal{L}} Q_{l}(t) x_{l} \\
\leq & U-\epsilon \alpha W_{s}(t) \bar{\lambda}-\epsilon \sum_{l \in \mathcal{L}} Q_{l}(t) x_{l} \\
\leq & -U,
\end{aligned}
$$

where inequality (14) holds due to the definition of $\epsilon_{1}$ (11), and inequality (15) holds due to inequality (12).

- Case III: Assume that $W_{s}(t) \leq U_{W}$ and $Q_{l}(t)>U_{Q}$ for some $l$. In this case, if a long-lived flow is selected for a given $\mathbf{c}$, we have

$$
\text { (10) } \leq \alpha W_{s}(t) \mu_{\mathbf{c}, s} \leq \alpha W_{s}(t) \text {. }
$$

Otherwise, if a short-lived flow is selected, it means for the given $\mathbf{c}$, we have $\max _{l} Q_{l}(t) R_{\mathbf{c}, l} \leq \alpha W_{s}(t)$, and

$$
\text { (10) } \leq 2 \alpha W_{s}(t) \text {. }
$$

Therefore, we can conclude that in this case,

$$
\begin{aligned}
& \mathbf{E}[V(t+1)-V(t) \mid \mathbf{M}(t)] \\
\leq & U+4 \alpha W_{s}(t)-2 \epsilon \alpha W_{s}(t) \bar{\lambda}-2 \epsilon \sum_{l \in \mathcal{L}} Q_{l}(t) x_{l} \\
\leq & U+4 \alpha U_{W}-2 \epsilon \alpha W_{s}(t) \bar{\lambda}-2 \epsilon \sum_{l \in \mathcal{L}} Q_{l}(t) x_{l} \\
\leq & -U
\end{aligned}
$$

where the last inequality yields from the definition of $U_{Q}$ (13).

From the analysis above, we can conclude that

$\mathbf{E}[V(t+1)-V(t) \mid \mathbf{M}(t)] \leq U_{d} 1_{\mathbf{M}(t) \in \Upsilon}-U 1_{\mathbf{M}(t) \notin \Upsilon}$,

where $U_{d}=U+2 \alpha U_{W}+2 R^{\max } L U_{Q}$ and $\Upsilon$ is a set with a finite number of elements. Invoking Foster's criterion, the Markov chain $\mathbf{M}(t)$ is positive recurrent and the theorem holds.

\section{Appendix B: Proof of TheOREM 3}

Consider the network that is operated under WSL, and define $\mathcal{H}(t)$ to be

$$
\mathcal{H}(t) \triangleq\left\{Q_{l}(t), R_{l}(t), Q_{i}(t), R_{i}(t), \tilde{R}_{i}^{\max }(t)\right\} .
$$

Now given $\mathcal{H}(t)$, we define the following notations:

- Define $\mu_{2 ; l}(t)=R_{l}(t)$ if flow $l$ is selected by WSL, and $\mu_{2 ; l}(t)=0$ otherwise.

- Define $\mu_{2 ; i}(t)=1$ if flow $i$ is selected by WSL and the workload of flow $i$ can be reduced by one, and $\mu_{2 ; i}(t)=0$ otherwise.

- Define $\mu_{1 ; l}(t)=R_{l}(t)$ if flow $l$ is selected by WS, and $\mu_{1 ; l}(t)=0$ otherwise. 
- Define $\mu_{1 ; i}(t)=1$ if flow $i$ is selected by WS and the workload of flow $i$ can be reduced by one, and $\mu_{1 ; i}(t)=0$ otherwise.

We remark that $\mu_{2 ; j}(t)$ is the action selected by the base station at time $t$ under WSL and $\mu_{1 ; j}(t)$ is the action selected by the base station at time $t$ under WS, assuming the same history $\mathcal{H}(t)$.

We define the Lyapunov function to be

$$
V(n)=\alpha\left(W_{s}(n T)\right)^{2}+\sum_{l \in \mathcal{L}}\left(Q_{l}(n T)\right)^{2} .
$$

First, it is easy to verify that there exists $U_{1}$ independent of $\tilde{\mathbf{M}}(n)$ such that

$$
\begin{aligned}
& \mathbf{E}[V(n+1)-V(n) \mid \tilde{\mathbf{M}}(n)] \\
&<U_{1}+2 \alpha \mathbf{E}\left[W_{s}(n T) \sum_{t=n T}^{(n+1) T-1}\left(A_{s}(t)-\mu_{2 ; s}(t)\right) \mid \tilde{\mathbf{M}}(n)\right] \\
&+\sum_{l \in \mathcal{L}} 2 \mathbf{E}\left[Q_{l}(n T) \sum_{t=n T}^{(n+1) T-1}\left(X_{l}(t)-\mu_{2 ; l}(t)\right) \mid \tilde{\mathbf{M}}(n)\right] .
\end{aligned}
$$

Dividing the time into two segments $[n T, n T+D-1]$ and $[n T+D,(n+1) T-1]$, we obtain

$$
\begin{aligned}
& \quad \mathbf{E}[V(n+1)-V(n) \mid \tilde{\mathbf{M}}(n)] \\
& <U_{1}+2 \alpha W_{s}(n T) \bar{\lambda} D+2 \sum_{l \in \mathcal{L}} Q_{l}(n T) x_{l} D \\
& +2 \alpha \mathbf{E}\left[W_{s}(n T) \sum_{t=n T+D}^{(n+1) T-1}\left(A_{s}(t)-\mu_{2 ; s}(t)\right) \mid \tilde{\mathbf{M}}(n)\right] \\
& +\sum_{l \in \mathcal{L}} 2 \mathbf{E}\left[Q_{l}(n T) \sum_{t=n T+D}^{(n+1) T-1}\left(X_{l}(t)-\mu_{2 ; l}(t)\right) \mid \tilde{\mathbf{M}}(n)\right] .
\end{aligned}
$$

Note that $\left|Q_{l}\left(t_{1}\right)-Q_{l}\left(t_{2}\right)\right|$ and $\left|W_{k}\left(t_{1}\right)-W_{k}\left(t_{2}\right)\right|$ are both bounded by some constants independent of $\tilde{\mathbf{M}}(n)$, so there exists $\tilde{U}$ such that

$$
\begin{aligned}
& \mathbf{E}[V(n+1)-V(n) \mid \tilde{\mathbf{M}}(n)] \\
& <\tilde{U}+2 \alpha W_{s}(n T) \bar{\lambda} D+2 \sum_{l \in \mathcal{L}} Q_{l}(n T) x_{l} D \\
& +2 \mathbf{E}\left[\alpha \sum_{t=n T+D}^{(n+1) T-1} W_{s}(t)\left(A_{s}(t)-\mu_{2 ; s}(t)\right) \mid \tilde{\mathbf{M}}(n)\right] \\
& +\sum_{l \in \mathcal{L}} 2 \mathbf{E}\left[\sum_{t=n T+D}^{(n+1) T-1} Q_{l}(t)\left(X_{l}(t)-\mu_{2 ; l}(t)\right) \mid \tilde{\mathbf{M}}(n)\right] .
\end{aligned}
$$

Now, by adding and subtracting $\mu_{1 ; \cdot}(t)$, we obtain

$$
\begin{aligned}
& \mathbf{E}[V(n+1)-V(n) \mid \tilde{\mathbf{M}}(n)] \\
\leq & \tilde{U}+2 \alpha W_{s}(n T) \bar{\lambda} D+2 \sum_{l \in \mathcal{L}} Q_{l}(n T) x_{l} D+\sum_{t=n T+D}^{(n+1) T-1} \operatorname{Drift}(t),
\end{aligned}
$$

where

$$
\begin{aligned}
& \operatorname{Drift}(t) \\
= & 2 \mathbf{E}\left[\alpha W_{s}(t) A_{s}(t)+\sum_{l \in \mathcal{L}} Q_{l}(t) X_{l}(t) \mid \tilde{\mathbf{M}}(n)\right] \\
& -2 \mathbf{E}\left[\alpha W_{s}(t) \mu_{1 ; s}(t)+\sum_{l \in \mathcal{L}} Q_{l}(t) \mu_{1 ; l}(t) \mid \tilde{\mathbf{M}}(n)\right] \\
& +\sum_{l \in \mathcal{L}} 2 \mathbf{E}\left[Q_{l}(t)\left(\mu_{1 ; l}(t)-\mu_{2 ; l}(t)\right) \mid \tilde{\mathbf{M}}(n)\right] \\
& +2 \mathbf{E}\left[\alpha W_{s}(t)\left(\mu_{1 ; s}(t)-\mu_{2 ; s}(t)\right) \mid \tilde{\mathbf{M}}(n)\right] .
\end{aligned}
$$

Note that 21 +22 is the difference between WS and WSL. In the following analysis, we will prove that this difference is small compared to the absolute value of $19+20)$.

We define

$$
\begin{aligned}
\operatorname{Diff}(t)= & \alpha W_{s}(t)\left(\mu_{1 ; s}(t)-\mu_{2 ; s}(t)\right) \\
& +\sum_{l \in \mathcal{L}} Q_{l}(t)\left(\mu_{1 ; l}(t)-\mu_{2 ; l}(t)\right),
\end{aligned}
$$

and compute its value in three different situations:

- Situ-A: Consider the situation in which $\alpha \tilde{W}_{s}(t) \leq$ $\max _{l \in \mathcal{L}} Q_{l}(t) R_{l}(t)$. We note that $\tilde{W}_{s}(t) \geq W_{s}(t)$ since $\tilde{R}_{i}^{\max }(t) \leq R_{i}^{\max }$ for all $t$ and $i$. Therefore, given $\alpha \tilde{W}_{s}(t) \leq \sum_{l \in \mathcal{L}} Q_{l}(t)$, both WS and WSL will select a long-lived flow. In this case, we can conclude that

$$
\mu_{1 ; l}(t)=\mu_{2 ; l}(t) \text { and } \mu_{1 ; s}(t)=\mu_{2 ; s}(t)=0,
$$

and

$$
\operatorname{Diff}(t)=0
$$

- Situ-B: Consider the situation in which $\alpha W_{s}(t)>$ $\max _{l \in \mathcal{L}} Q_{l}(t) R_{l}(t)$. In this case, both WS and WSL will select a short-lived flow, which implies that

$$
\mu_{1 ; l}(t)=\mu_{2 ; l}(t)=0,
$$

and

$$
\begin{aligned}
\operatorname{Diff}(t)= & \alpha W_{s}(t)\left(\mu_{1 ; s}(t)-\mu_{2 ; s}(t)\right) \\
& \leq \alpha W_{s}(t)\left(1-\mu_{2 ; s}(t)\right)
\end{aligned}
$$

- Situ-C: Consider the situation in which $\alpha \tilde{W}_{s}(t)>$ $\max _{l \in \mathcal{L}} Q_{l}(t) R_{l}(t) \geq \alpha W_{s}(t)$. In this case, WS will select a long-lived flow and WSL will select a short-lived flow. We hence have

$$
\mu_{1 ; l}(t)>0 \text { and } \mu_{1 ; s}(t)=\mu_{2 ; l}(t)=0,
$$

and

$$
\begin{aligned}
\operatorname{Diff}(t) & =\max _{l \in \mathcal{L}} Q_{l}(t) R_{l}(t)-\alpha W_{s}(t) \mu_{2 ; s}(t) \\
& \leq \alpha \tilde{W}_{s}(t)-\alpha W_{s}(t) \mu_{2 ; s}(t)
\end{aligned}
$$


According to the analysis above, we have that

$$
\begin{aligned}
& \mathbf{E}[\operatorname{Diff}(t) \mid \tilde{\mathbf{M}}(n)] \\
\leq & \mathbf{E}\left[\alpha W_{s}(t) \mid \operatorname{Situ}-\mathbf{B}, \mu_{2 ; s}=0, \tilde{\mathbf{M}}(n)\right] \times \\
& \operatorname{Pr}\left(\operatorname{Situ}-\mathbf{B}, \mu_{2 ; s}=0 \mid \tilde{\mathbf{M}}(n)\right) \\
+ & \mathbf{E}\left[\alpha \tilde{W}_{s}(t) \mid \operatorname{Situ}-\mathrm{C}, \mu_{2 ; s}=0, \tilde{\mathbf{M}}(n)\right] \times \\
& \operatorname{Pr}\left(\operatorname{Situ}-\mathrm{C}, \mu_{2 ; s}=0 \mid \tilde{\mathbf{M}}(n)\right) \\
+ & \mathbf{E}\left[\alpha \tilde{W}_{s}(t)-\alpha W_{s}(t) \mid \operatorname{Situ}-\mathbf{C}, \mu_{2 ; s}=1, \tilde{\mathbf{M}}(n)\right] \times \\
& \operatorname{Pr}\left(\operatorname{Situ}-\mathbf{C}, \mu_{2 ; s}=1 \mid \tilde{\mathbf{M}}(n)\right) .
\end{aligned}
$$

Next we define a finite set $\tilde{\Upsilon}$. We first introduce some constants:

- $\epsilon_{1}=\min \left\{\frac{\bar{\lambda} \epsilon}{16}, \frac{\epsilon \min _{l} x_{l}}{4 R^{\max }}\right\}$.

- $\epsilon_{2}=\frac{\bar{\lambda} \epsilon}{16 R^{\max }}$, and $D_{\epsilon_{2}}$ and $N_{\epsilon_{2}}$ are the numbers that guarantee $\operatorname{Pr}\left(\mathcal{E}_{\text {miss }}(t)\right) \leq \epsilon_{2}$, which are defined by the goodness of the tie-breaking rule.

- $\lambda_{W}^{\max }=K \lambda^{\max } F^{\max }$, which is the maximum number of bits of short-lived flows injected in one time slot, and also the upper bound on the new workload injected in the network in one time slot.

We define a set $\tilde{\Upsilon}$ such that

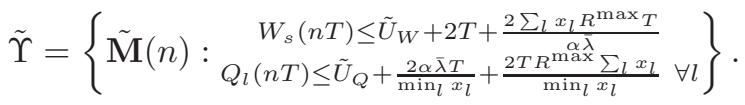

In this definition, $\tilde{U}_{W}$ is a positive integer satisfying that

$$
\begin{gathered}
\left(1-p_{s}^{\max }\right) \frac{\tilde{U}_{W}}{F^{\max }} \leq \epsilon_{1} \\
\tilde{U}_{W} \geq \frac{\frac{4 \tilde{U}}{T-D}+8 \epsilon_{2} \alpha \lambda_{W}^{\max } T+4 \alpha D R^{\max }+8 \epsilon_{2} \alpha R^{\max } T+4 \lambda_{W}^{\max } D}{\epsilon \alpha \bar{\lambda}} \\
\frac{\tilde{U}_{W}}{F^{\max }} \geq N_{\epsilon_{2}}
\end{gathered}
$$

and $\tilde{U}_{Q}$ is a positive integer satisfying

$$
\tilde{U}_{Q} \geq \frac{4 \tilde{U}+6 \alpha R^{\max }\left(\tilde{U}_{W}+\frac{2 \sum_{l} x_{l} R^{\max } T}{\alpha \lambda}+\left(\lambda_{W}^{\max }+2\right) T\right)}{\epsilon \min _{l} x_{l}} .
$$

Since the changes of $W_{s}(t)$ and $Q_{l}(t)$ during each time slot is bounded by some constants independent of $\tilde{\mathbf{M}}(n)$, it is easy to verify that $\tilde{\Upsilon}$ is a set of a finite number of elements.

Next, we analyze the drift of Lyapunov function case by case assuming that $D>\left\lceil\frac{\log \bar{\lambda} \epsilon-\log 16-\log R^{\max }}{\log \left(1-p_{s}^{\max }\right)}\right\rceil$ and $T>$ $\left\lceil\frac{(4+\epsilon) D}{\epsilon}\right\rceil$.

- Case I: Assume that $\tilde{M}(n) \in \tilde{\Upsilon}$. In this case, it is easy to verify that $\mathbf{E}[V(n+1)-V(n) \mid \tilde{\mathbf{M}}(n)]$ is bounded by some constant $\tilde{U}_{d}$.

- Case II: Assume that

$$
W_{s}(n T)>\tilde{U}_{W}+2 T+\frac{2 \sum_{l} x_{l} R^{\max } T}{\alpha \bar{\lambda}} \geq \tilde{U}_{W}+T .
$$

Recall that $\mathcal{E}_{\text {miss }}(t)$ is the event such that the tie-breaking rule selects a short-lived flow with $\tilde{R}_{i}^{\max }(t) \neq R_{i}^{\max }$. Note that $\mu_{2 ; s}(t)=0$ implies that $\mathcal{E}_{\text {miss }}(t)$ occurs. Also note the following facts:
- For any $n T \leq t \leq(n+1) T$, we have $W(t) \leq$ $W(n T)+\lambda_{W}^{\max } T$,

- Given $W_{s}(n T) \geq \tilde{U}_{W}+T$, we have $W_{s}(t) \geq \tilde{U}_{W}$ for all $n T \leq t \leq(n+1) T-1$. Then according to the definition of $\epsilon_{2}$ and $\tilde{U}_{W}$ and assumption that the tie-breaking rule is good, we have

$$
\operatorname{Pr}\left(\mathcal{E}_{\text {miss }}(t)\right) \leq \epsilon_{2}
$$

for all $n T+D \leq t \leq(n+1) T-1$.

- Given any $\tilde{\mathbf{M}}(n)$ and any $n T+D \leq t \leq(n+1) T-1$, we have

$$
\begin{aligned}
& \mathbf{E}\left[\alpha \tilde{W}_{s}(t)-W_{s}(t) \mid \text { Situ-C, } \mu_{2 ; s}=1, \tilde{\mathbf{M}}(n)\right] \times \\
& \operatorname{Pr}\left(\text { Situ-C, } \mu_{2 ; s}=1 \mid \tilde{\mathbf{M}}(n)\right) \\
\leq & \mathbf{E}\left[\alpha \tilde{W}_{s}(t)-W_{s}(t) \mid \tilde{\mathbf{M}}(n)\right] \\
= & \mathbf{E}\left[\mathbf{E}\left[\alpha \tilde{W}_{s}(t)-\alpha W_{s}(t)\left|W_{s}(t-D)\right|\right] \mid \tilde{\mathbf{M}}(n)\right] \\
\leq & \mathbf{E}\left[\alpha\left(1-p_{s}^{\max }\right)^{D} W_{s}(t-D) R^{\max }+\alpha \lambda_{W}^{\max } D \mid \tilde{\mathbf{M}}(n)\right]
\end{aligned}
$$

$\leq \mathbf{E}\left[\alpha\left(1-p_{s}^{\max }\right)^{D}\left(W_{s}(t)+D\right) R^{\max }+\alpha \lambda_{W}^{\max } D \mid \tilde{\mathbf{M}}(n)\right]$,

where the inequality 27 holds because at most $\lambda_{W}^{\max } D$ bits belonging to short-lived flows are in the network for less than $D$ time slots at time $t$, and a flow having been in the network for at least $D$ time slots can estimate correctly its workload with a probability at least 1 $\left(1-p_{s}^{\max }\right)^{D}$.

Now according to the observations above, we can obtain that

$$
\begin{aligned}
& \mathbf{E}[\operatorname{Diff}(t) \mid \tilde{\mathbf{M}}(n)] \\
\leq & \epsilon_{2} \alpha\left(W_{s}(n T)+\lambda_{W}^{\max } T\right)+\epsilon_{2} \alpha\left(R^{\max } W_{s}(n T)+\lambda_{W}^{\max } T\right) \\
& +\mathbf{E}\left[\alpha\left(1-p_{s}^{\max }\right)^{D}\left(W_{s}(t)+D\right) R^{\max }+\alpha \lambda_{W}^{\max } D \mid \tilde{\mathbf{M}}(n)\right] .
\end{aligned}
$$

Combining with the analysis leading to (14) in Appendix A, we conclude that

$$
\begin{aligned}
& \quad \operatorname{Drift}(t) \\
& \leq 2 \mathbf{E}\left[\epsilon_{1}\left(\alpha W_{s}(t)+R^{\max } \max _{l \in \mathcal{L}} Q_{l}(t)\right)\right. \\
& \quad-\epsilon \alpha W_{s}(t) \bar{\lambda}-\epsilon \sum_{l \in \mathcal{L}} Q_{l}(t) x_{l} \\
& \quad+\epsilon_{2} \alpha\left(W_{s}(n T)+\lambda_{W}^{\max } T\right) \\
& \quad+\epsilon_{2} \alpha\left(R^{\max } W_{s}(n T)+\lambda_{W}^{\max } T\right) \\
& \left.+\alpha\left(1-p_{s}^{\max }\right)^{D}\left(W_{s}(t)+D\right) R^{\max }+\alpha \lambda_{W}^{\max } D \mid \tilde{\mathbf{M}}(n)\right] \\
& \leq \mathbf{E}\left[-\epsilon\left(\alpha \bar{\lambda} W_{s}(t)+\sum_{l \in \mathcal{L}} x_{l} Q_{l}(t)\right) \mid \tilde{\mathbf{M}}(n)\right],
\end{aligned}
$$

where the last inequality holds due to the definition of $\tilde{U}_{W}$.

- Case III: Assume that

$$
W_{s}(n T)<\tilde{U}_{W}+2 T+\frac{2 \sum_{l} x_{l} R^{\max } T}{\alpha \bar{\lambda}}
$$


and

$$
Q_{l}(n T)>\tilde{U}_{Q}+\frac{2 \alpha \bar{\lambda} T}{\min _{l} x_{l}}+\frac{2 T R^{\max } \sum_{l} x_{l}}{\min _{l} x_{l}}>\tilde{U}_{Q}
$$

for some $l$. In this case, we have

$$
\operatorname{Diff}(t) \leq \alpha \tilde{W}_{s}(t) \leq \alpha R^{\max } W_{s}(t) .
$$

Combining with the analysis leading to 16 in Appendix A, we have that

$$
\begin{aligned}
& \operatorname{Drift}(t) \\
\leq & 2 \mathbf{E}\left[\alpha R^{\max } W_{s}(t)+2 \alpha W_{s}(t)\right. \\
& \left.-\epsilon\left(\alpha \bar{\lambda} W_{s}(t)+\sum_{l \in \mathcal{L}} x_{l} Q_{l}(t)\right) \mid \tilde{\mathbf{M}}(n)\right] \\
\leq & \mathbf{E}\left[-\epsilon\left(\alpha \bar{\lambda} W_{s}(t)+\sum_{l \in \mathcal{L}} x_{l} Q_{l}(t)\right) \mid \tilde{\mathbf{M}}(n)\right],
\end{aligned}
$$

where the last inequality holds due to the definition of $\tilde{U}_{Q}$.

Now, combining the three cases together, we can obtain that

$$
\begin{aligned}
& \quad \mathbf{E}[V(n+1)-V(n) \mid \tilde{\mathbf{M}}(n)] \\
& \leq \tilde{U}+2 \alpha W_{s}(n T) \bar{\lambda} D+2 \sum_{l \in \mathcal{L}} Q_{l}(n T) x_{l} D \\
& \quad+\sum_{t=n T+D}^{(n+1) T-1} \mathbf{E}\left[-\epsilon\left(\alpha \bar{\lambda} W_{s}(t)+\sum_{l \in \mathcal{L}} x_{l} Q_{l}(t)\right) \mid \tilde{\mathbf{M}}(n)\right] \\
& \leq \tilde{U}+2 \alpha W_{s}(n T) \bar{\lambda} D+2 \sum_{l \in \mathcal{L}} Q_{l}(n T) x_{l} D \\
& \quad-\epsilon(T-D)\left(\alpha \bar{\lambda} W_{s}(n T)+\sum_{l \in \mathcal{L}} x_{l} Q_{l}(n T)\right) \\
& \quad+\epsilon(T-D)\left(\alpha \bar{\lambda} T+\sum_{l \in \mathcal{L}} x_{l} R^{\max T)}\right. \\
& \leq-\tilde{U}
\end{aligned}
$$

where the last inequality yields from the definition of $\tilde{U}_{W}$ and $\tilde{U}_{Q}$. Finally, we can conclude the theorem from the Foster's Criterion.

\section{Appendix C: The Uniform TIE-BREAKIng Rule}

Recall that we define $\mathcal{E}_{\text {miss }}(t)$ to be the event that the tiebreaking rule selects a short-lived flow with $\tilde{R}_{i}^{\max }(t) \neq R_{i}^{\max }$.

Proposition 4: The uniform tie-breaking rule is good.

Proof: Suppose set

$$
\mathcal{J}(t)=\left\{i: R_{i}(t)=\tilde{R}_{i}^{\max }(t) \text { or } R_{i}(t) \geq Q_{i}(t)\right\} .
$$

Under the uniform tie-breaking, $\mathcal{E}_{m i s s}(t)$ occurs with probability

$$
\begin{aligned}
& \frac{\mid\left\{i: i \in \mathcal{J}(t) \text { and } \tilde{R}_{i}^{\max }(t) \neq R_{i}^{\max }\right\} \mid}{|\mathcal{J}(t)|} \\
\leq & \frac{\left|\left\{i: \tilde{R}_{i}^{\max }(t) \neq R_{i}^{\max }\right\}\right|}{\left|\left\{i: R_{i}(t)=\tilde{R}_{i}^{\max }(t)\right\}\right|} .
\end{aligned}
$$

Assume that $N$ short-lived flows are in the network at time $t-D$ and denote by $\mathcal{N}$ the set of these short-lived flows. Our proof contains the following two steps:

Step 1: We first obtain an upper bound on

$$
N_{1} \triangleq \mid\left\{i: \tilde{R}_{i}^{\max }(t) \neq R_{i}^{\max } \text { and } i \in \mathcal{N}\right\} \mid \text {. }
$$

Considering a short-lived flow (flow $i$ ) which is in the network at time $t-D$, we have

$$
\operatorname{Pr}\left(\tilde{R}_{i}^{\max }(t) \neq R_{i}^{\max }\right) \leq\left(1-p_{s}^{\max }\right)^{D} \triangleq \epsilon .
$$

Thus, $\mathbf{E}\left[N_{1}\right] \leq \epsilon N$. According to the Chernoff bound, we have

$$
\begin{aligned}
& \operatorname{Pr}\left(N_{1} \geq 1.1 \epsilon N+D\right) \\
\leq & \exp \left(-\frac{\left(1.1 \epsilon N+D-\mathbf{E}\left[N_{1}\right]\right)^{2}}{3 \mathbf{E}\left[N_{1}\right]}\right) \\
\leq & \exp \left(-\frac{(0.1 \epsilon N+D)^{2}}{3 \epsilon N}\right) \\
\leq & \exp (-0.003 \epsilon N+0.06 D) .
\end{aligned}
$$

Next note that at most $K \lambda^{\max }$ short-lived flows join the network during each time slot, so we can conclude that

$$
\begin{gathered}
\operatorname{Pr}\left(\left|\left\{i: \tilde{R}_{i}^{\max }(t) \neq R_{i}^{\max }\right\}\right| \geq 1.1 \epsilon N+K \lambda^{\max } D+D\right) \\
\leq \exp (-0.003 \epsilon N+0.06 D) .
\end{gathered}
$$

Step 2: Since at most one flow can be completely transmitted in one time slot, so least $N-D$ flows are in the network at time $t$, each having a probability at least $p_{s}^{\max }$ to be in the best channel state.

$$
\begin{aligned}
& \operatorname{Pr}\left(\left|\left\{i: R_{i}(t)=\tilde{R}_{i}^{\max }(t)\right\}\right| \leq 0.9 p_{s}^{\max }(N-D)\right) \\
\leq & \operatorname{Pr}\left(\left|\left\{i: R_{i}(t)=R_{i}^{\max }(t)\right\}\right| \leq 0.9 p_{s}^{\max }(N-D)\right) \\
\leq & \exp \left(-0.003 p_{s}^{\max }(N-D)\right) .
\end{aligned}
$$

Summary: From step 1 and step 2, we can conclude that

$$
\begin{aligned}
& \operatorname{Pr}\left(\mathcal{E}_{\text {miss }}(t)\right) \\
& \leq \frac{1.1 \epsilon N+K \lambda^{\max } D+D}{0.9 p_{s}^{\max }(N-D)}+\exp \left(-0.003 p_{s}^{\max }(N-D)\right) \\
& \quad+\exp (-0.003 \epsilon N+0.06 D),
\end{aligned}
$$

which converges to zero as both $D$ and $N / D$ go to infinity. The proposition holds because the sizes of short-lived flows are bounded and a large workload implies a large number of short-lived flows.

\section{APPENDix D: OldEST-FIRST TIE-BREAKING RUlE}

Proposition 5: The oldest-first tie-breaking is a good tiebreaking rule.

Proof: We assume that at time slot $t-D$, there are $N>$ $2 D^{2}$ short-lived flows in the network. We group short-lived flows into groups $\mathcal{G}$ according to the time they arrived at the network such that group $\mathcal{G}_{\bar{\tau}}(t)$ contains all flows arriving no less than $\bar{\tau}$ time slots ago at time $t$, and group $\mathcal{G}_{\tau}(t)$ contains the flows arriving exact $\tau$ time slots ago at time $t(\tau<\bar{\tau})$. 
Case 1: Assume that $\left|\mathcal{G}_{\bar{\tau}}(t-D)\right| \geq D^{2}$. We first consider the following probability

$\operatorname{Pr}\left(\right.$ a flow $\in \mathcal{G}_{\bar{\tau}}(t)$ is selected at $t$ and $\left.\tilde{R}_{i}^{\max }(t) \neq R_{i}^{\max }\right)$.

Note that $\mathcal{G}_{\bar{\tau}}(t)$ can contain at most $K \lambda^{\max } D$ additional flows compared to $\mathcal{G}_{\bar{\tau}}(t-D)$ since $\left|\mathcal{G}_{\tau}\right| \leq K \lambda^{\max }$ for all $\tau<\bar{\tau}$. Following the analysis for the uniform tie-breaking in Appendix $\mathrm{C}$, we can easily prove that

$$
\begin{aligned}
& \operatorname{Pr}\left(\text { a flow } \in \mathcal{G}_{\bar{\tau}}(t) \text { is selected at } t \text { and } \tilde{R}_{i}^{\max }(t) \neq R_{i}^{\max }\right) \\
& \rightarrow 0
\end{aligned}
$$

as $D$ goes to infinity.

Next, note that at most one short-lived flow can be completely transmitted in one time slot, so $\mathcal{G}_{\bar{\tau}}(t)$ containing at least $D^{2}-D$ flows at time $t$, which implies that

$$
\begin{aligned}
& \operatorname{Pr}\left(\text { a flow } \notin \mathcal{G}_{\bar{\tau}}(t) \text { is selected at } t \text { and } \tilde{R}_{i}^{\max }(t) \neq R_{i}^{\max }\right) \\
\leq & \left(1-p_{s}^{\max }\right)^{D^{2}-D} .
\end{aligned}
$$

Therefore, we conclude that

$$
\begin{aligned}
& \operatorname{Pr}\left(\text { the selected flow at } t \text { has } \tilde{R}_{i}^{\max }(t) \neq R_{i}^{\max }\right) \\
= & \operatorname{Pr}\left(\text { a flow } \mathcal{G}_{\bar{\tau}}(t) \text { is selected at } t \text { and } \tilde{R}_{i}^{\max }(t) \neq R_{i}^{\max }\right) \\
+ & \operatorname{Pr}\left(\text { a flow } \notin \mathcal{G}_{\bar{\tau}}(t) \text { is selected at } t \text { and } \tilde{R}_{i}^{\max }(t) \neq R_{i}^{\max }\right)
\end{aligned}
$$

which converges to zero as $D$ goes to infinity.

Case 2: Assume that $\left|\mathcal{G}_{\bar{\tau}}(t-D)\right|<D^{2}$. In this case, we search the groups starting from group $\mathcal{G}_{\bar{\tau}}(t)$ and stop at group $\tau^{*}$ if $D^{2}+D K \lambda^{\max }>\sum_{\tau=\tau^{*}}^{\bar{\tau}}\left|\mathcal{G}_{\tau}(t)\right| \geq D^{2}$. Note that when $D$ is sufficiently large, such $\tau^{*}$ exists and $\tau^{*}>D$ because $N>2 D^{2}$ and $\left|\mathcal{G}_{\tau}\right| \leq K \lambda^{\max }$ for all $\tau<\bar{\tau}$. Considering a certain flow $i$ such that $i \in \cup_{\tau=\tau^{*}}^{\bar{\tau}} \mathcal{G}_{\tau}(t)$, we have that

$$
\begin{aligned}
& \operatorname{Pr}\left(\text { flow } i \text { is selected at } t \text { and } \tilde{R}_{i}^{\max }(t) \neq R_{i}^{\max }\right) \\
& \leq \operatorname{Pr}\left(\tilde{R}_{i}^{\max }(t) \neq R_{i}^{\max }\right) \\
& \leq\left(1-p_{s}^{\max }\right)^{D} \triangleq \epsilon .
\end{aligned}
$$

Thus, we can obtain that

$$
\begin{aligned}
& \operatorname{Pr}\left(\text { a flow } \in \cup_{\tau=\tau^{*}}^{\bar{\tau}} \mathcal{G}_{\tau}(t) \text { is selected at } t \text { and } \tilde{R}_{i}^{\max }(t) \neq R_{i}^{\max }\right) \\
\leq & \left(D^{2}+D K \lambda^{\max }\right) \epsilon,
\end{aligned}
$$

which converges to zero as $D$ goes to infinity. Further, similar to the analysis in Case 1, we can obtain that when $D$ is sufficiently large,

$$
\begin{aligned}
& \operatorname{Pr}\left(\text { a flow } \notin \cup_{\tau=0}^{\tau^{*}-1} \mathcal{G}_{h}(t) \text { is selected at } t \text { and } \tilde{R}_{i}^{\max }(t) \neq R_{i}^{\max }\right) \\
\leq & \left(1-p_{s}^{\max }\right)^{D^{2}}
\end{aligned}
$$

which converges to zero as well.

Combining Case 1 and 2, we can conclude that the oldestfirst tie-breaking is a good tie-breaking rule.

\section{REFERENCES}

[1] P. van de Ven, S. Borst, and S. Shneer, "Instability of MaxWeight scheduling algorithms," in Proc. IEEE Infocom., Rio de Janeiro, Brazil, April 2009, pp. 1701 - 1709.

[2] L. Tassiulas and A. Ephremides, "Stability properties of constrained queueing systems and scheduling policies for maximum throughput in multihop radio networks", IEEE Transactions on Automatic Control, Vol. 37, No. 12, pp. 1936-1949, December 1992.

[3] M. Andrews, K. Kumaran, K. Ramanan, A. Stolyar, R. Vijayakumar, and P. Whiting, "Scheduling in a queueing system with aynchronously varying service rates," Probability in the Engineering and Informational Sciences, vol. 18, pp. 191-217, 2004.

[4] A. Eryilmaz, R. Srikant, and J. R. Perkins, "Stable scheduling policies for fading wireless channels," IEEE/ACM Trans. Network., vol. 13, no. 2, pp. 411-424, 2005.

[5] M. J. Neely, E. Modiano, and C. E. Rohrs, "Dynamic Power Allocation and Routing for Time Varying Wireless Networks," IEEE Journal on Selected Areas in Communications, Special Issue on Wireless Ad-Hoc Networks, vol. 23, no. 1, pp. 89-103, Jan. 2005.

[6] X. Liu, E. Chong, and N. Shroff, "Opportunistic Transmission Scheduling with Resource-Sharing Constraints in Wireless Networks," IEEE Journal on Selected Areas in Communications, vol. 19, no. 10, pp. 20532064, October, 2001.

[7] P. Viswanath, D. Tse and R. Laroia, "Opportunistic Beamforming using Dumb Antennas," IEEE Transactions on Information Theory, Vol. 48, No. 6, pp. 1277-1294, June 2002.

[8] X. Lin, N. B. Shroff and R. Srikant, "A Tutorial on Cross-Layer Optimization in Wireless Networks," in IEEE Journal on Selected Areas in Communications, vol. 24, no. 8, August 2006.

[9] L. Georgiadis, M. Neely and L. Tassiulas, Resource Allocation and Cross Layer Control in Wireless Networks, NoW publishers, 2006.

[10] S. Shakkottai and R. Srikant, Network Optimization and Control. NoW publishers, 2007.

[11] S. Asmussen. Applied Probability and the Theory of Queues. Springer, 2003. 